\title{
Classification of finite energy solutions to the fractional Lane-Emden-Fowler equations with slightly subcritical exponents
}

\author{
Woocheol Choi ${ }^{1}$ - Seunghyeok Kim ${ }^{2}$
}

Received: 31 October 2015 / Accepted: 9 April 2016 / Published online: 26 April 2016

(C) Fondazione Annali di Matematica Pura ed Applicata and Springer-Verlag Berlin Heidelberg 2016

\begin{abstract}
We study qualitative properties of solutions to the fractional Lane-Emden-Fowler equations with slightly subcritical exponents where the associated fractional Laplacian is defined in terms of either the spectra of the Dirichlet Laplacian or the integral representation. As a consequence, we classify the asymptotic behavior of all finite energy solutions. Our method also provides a simple and unified approach to deal with the classical (local) LaneEmden-Fowler equation for any dimension $>2$.
\end{abstract}

Keywords Fractional Laplacian · Critical nonlinearity · Multi-peak solutions · Blow-up analysis

Mathematics Subject Classification Primary 35R11; Secondary 35B33 · 35B44 · 35B45 . 35J08

\section{Contents}

1 Introduction . . . . . . . . . . . . . . . . . . . . . . . . 270

2 Preliminaries on fractional Laplacians . . . . . . . . . . . . . . . . . . . . . . . . . . . . . 276

2.1 Definition of Sobolev spaces and fractional Laplacians . . . . . . . . . . . . . . . . . . 276

2.2 Localization of fractional Laplacians . . . . . . . . . . . . . . . . . . . . . . . . . . . . . 277

2.3 Green's functions of fractional Laplacians . . . . . . . . . . . . . . . . . . . . . . . . . . . 278

2.4 Sharp Sobolev and trace inequalities . . . . . . . . . . . . . . . . . . . . . . . . . . . . 279

2.5 Concentration-compactness principle . . . . . . . . . . . . . . . . . . . . . 280

3 Moving sphere argument and pointwise upper bound . . . . . . . . . . . . . . . . . . . . . . . 282

4 Application of the pointwise upper estimate . . . . . . . . . . . . . . . . . . . . . . . . . . . 287

5 Proof of main theorems for the spectral fractional Laplacian . . . . . . . . . . . . . . . . . . . . . 294

6 The restricted fractional Laplacian and the classical Laplacian . . . . . . . . . . . . . . . . . . . . 300

Seunghyeok Kim

shkim0401@gmail.com

1 School of Mathematics, Korea Institute for Advanced Study, Seoul 130-722, Republic of Korea

2 Facultad de Matemáticas, Pontificia Universidad Católica de Chile, Avenida Vicuña Mackenna 4860, Santiago, Chile 
6.1 Proof of Theorems 1.1 and 1.3 for the restricted fractional Laplacian . . . . . . . . . . . . . 300

6.2 Proof of Theorem B . . . . . . . . . . . . . . . . . . . . . . . . . . . 300

7 Appendix 1: Lower and upper estimates of the standard bubble in $\mathbb{R}_{+}^{N+1} \ldots \ldots \ldots$

8 Appendix 2: Elliptic regularity results and derivation of (4.22) and (4.23) . . . . . . . . . . . . . 303

References . . . . . . . . . . . . . . . . . . . . . . 306

\section{Introduction}

Suppose that $s \in(0,1), N>2 s, p=\frac{N+2 s}{N-2 s}$ and $\Omega$ is a smooth open bounded domain. In this paper, we are concerned with the asymptotic behavior of solutions to the nonlinear nonlocal elliptic problem

$$
\begin{cases}(-\Delta)^{s} u=u^{p-\epsilon} & \text { in } \Omega, \\ u>0 & \text { in } \Omega, \\ u=0 & \text { in } \mathbb{R}^{N} \backslash \bar{\Omega},\end{cases}
$$

when a small parameter $\epsilon>0$ tends to zero. Here, $(-\Delta)^{s}$ is understood as either the spectral fractional Laplacian or the restricted fractional Laplacian (see Sect. 2.1 for the definition of the fractional Laplacians).

Recently, various nonlocal differential equations have attracted lots of researchers. In particular, equations involving the fractional Laplacian were treated extensively in both pure and applied mathematics, because not only the fractional Laplacian is an operator which naturally interpolates the classical Laplacian $-\Delta$ and the identity $(-\Delta)^{0}=i d$, but also it appears in diverse areas including physics, biological modeling and mathematical finances, as a tool describing nonlocal characteristic.

Owing to technical difficulties arising from the nonlocality, there had not been enough progress in theory of equations involving the fractional Laplacian. However, about a decade ago, Caffarelli and Silvestre [15] interpreted the fractional Laplacian in $\mathbb{R}^{N}$ in terms of a Dirichlet-Neumann-type operator in the extended domain $\mathbb{R}_{+}^{N+1}=\left\{(x, t) \in \mathbb{R}^{N+1}: t>0\right\}$, and this idea allowed one to analyze nonlocal problems by utilizing well-known arguments such as the mountain pass theorem, the moving plane method, the Moser iteration technique or monotonicity formulae. A similar extension was also devised by Cabré and Tan [14], and Stinga and Torrea [58] (see Capella et al. [17], Brändle et al. [10], Tan [61] and Chang and González [19] also) for nonlocal elliptic equations on bounded domains with zero Dirichlet boundary condition.

Based on these extensions (or the integral representation of a differential operator itself), a lot of studies on nonlocal problems of the form $(-\Delta)^{s} u=f(u)$ (for a certain function $f: \mathbb{R} \rightarrow \mathbb{R}$ ) were conducted. For the results of particular equations, we refer to papers on the Schrödinger equations [3,22,28,32], the Allen-Cahn equations [12,13], the Fisher-KPP equations [8,11], the Nirenberg problem [1,39,40], and the Yamabe problem [24,34,35,41], respectively. Also, Brezis-Nirenberg-type problems have been tackled in $[6,27,60]$. Most results mentioned here considered on the existence of solutions with some desired property. Meanwhile, several regularity results such as the Schauder estimate and the strong maximum principle were derived in $[12,14,16,17,39,58]$ and references therein.

Due to its simple form, the Lane-Emden-Fowler problem (1.1) has been regarded as one of the most fundamental nonlinear elliptic equations. It is now a classical fact that the exponent $p=\frac{N+2 s}{N-2 s}$ is a threshold on the existence of a solution to (1.1). If $\epsilon>0$, one can find a solution to (1.1) by applying the standard variational argument with the compact embedding $H^{s}(\Omega) \hookrightarrow L^{p+1-\epsilon}(\Omega)$. If $\epsilon \leq 0$ and $\Omega$ is star-shaped, the Pohozaev identity 
(obtained in [14,61] for the spectral Laplacians and in [54] for the restricted Laplacians) implies that no solution exists. In view of the corresponding result of Bahri and Coron [4] to the case $s=1$, it is expected that (1.1) has a solution if the domain $\Omega$ has nontrivial topology.

On the other hand, it is well known that the Brezis-Nirenberg-type problem

$$
\begin{cases}(-\Delta)^{s} u=u^{p}+\epsilon u^{q} & \text { in } \Omega, \\ u>0 & \text { in } \Omega, \\ u=0 & \text { in } \mathbb{R}^{N} \backslash \bar{\Omega},\end{cases}
$$

where $N>2 s, 0<q<p$ and $\epsilon>0$ is a parameter, shares many common characteristics with (1.1). Through the papers $[6,7,57,60]$, it was determined that its solvability relies on $\epsilon, p, q, N$ and $\Omega$.

Once the existence theory is settled, the very next step would be to obtain information on the shape of solutions.

For Eq. (1.1) with general exponents on the nonlinearity, an answer of this question is provided by the moving plane argument. It yields that for any $p-\epsilon>1$ each solution to (1.1) increases along lines emanating from a boundary point to a certain interior point. It then induces symmetry of a solution from that of the domain $\Omega$. We refer to $[14,52,61]$ for further discussion.

On the other hand, it is natural to guess that if $\epsilon \rightarrow 0$, then the solution $u_{\epsilon}$ may possess a singular behavior, since $p=\frac{N+2 s}{N-2 s}$ is the critical exponent. This idea intrigues one to investigate the shape of $u_{\epsilon}$ in detail for $\epsilon>0$ small enough. In this regard, Choi et al. [25] and Dávila et al. [29] constructed multiple blow-up solutions by applying the Lyapunov-Schmidt reduction method (refer to Theorem A below). When the fractional Laplacian is defined in terms of the spectra of the Dirichlet Laplacian, the authors of [25] also characterized the asymptotic behavior of a sequence $\left\{u_{\epsilon}\right\}_{\epsilon>0}$ of minimal energy solutions to (1.1) and (1.2) (with $q=1$ ). It turned out that $u_{\epsilon}$ blows up at a single point which is a critical point of the Robin function of $(-\Delta)^{s}$.

In this line of research, an important remaining problem is to study the asymptotic character of solutions $\left\{u_{\epsilon}\right\}_{\epsilon>0}$ without the minimal energy condition. This is what we address in the current paper. Precisely, we shall give a detailed description for the asymptotic behavior of all finite energy solutions to (1.1) where the fractional Laplacian is either spectral or restricted one. We believe that the same phenomena should happen to finite energy solutions to (1.2).

Theorem 1.1 For any given $s \in(0,1)$ and $N>2 s$, suppose that there exists a sequence $\left\{u_{n}\right\}_{n \in \mathbb{N}}$ in

$$
\widetilde{H}^{s}(\Omega):=\left\{u \in H^{s}\left(\mathbb{R}^{N}\right): u=0 \text { in } \mathbb{R}^{N} \backslash \bar{\Omega}\right\}
$$

such that each of the function $u_{n}$ solves Eq. (1.1) with $\epsilon=\epsilon_{n} \searrow 0$. In addition, assume $\sup _{n \in \mathbb{N}}\left\|u_{n}\right\|_{\widetilde{H}^{s}(\Omega)}<+\infty$ (refer to Sect. 2.1). Then, one of the following holds: Up to a subsequence, either

(1) the function $u_{n}$ converges strongly in $\widetilde{H}^{s}(\Omega)$ to a function $v$ satisfying

$$
\begin{cases}(-\Delta)^{s} v=v^{p} & \text { in } \Omega, \\ v>0 & \text { in } \Omega, \\ v=0 & \text { on } \mathbb{R}^{N} \backslash \bar{\Omega}\end{cases}
$$

as $n \rightarrow \infty$, or 
(2) the asymptotic behavior of $u_{n}$ is given by

$$
u_{n}=\sum_{i=1}^{m} P w_{\lambda_{n}^{i}, x_{n}^{i}}+r_{n}
$$

where $\lambda_{n}^{i} \rightarrow 0$ and $x_{n}^{i} \rightarrow x_{0}^{i} \in \Omega$ as $n \rightarrow \infty$. Here, $P w_{\lambda, \xi}$ is the projected bubble defined after (2.17), and $r_{n}$ is a remainder term converging to zero in $\widetilde{H}^{s}(\Omega)$. Furthermore, the following properties are valid.

- There is a constant $C_{0}>0$ independent of $n \in \mathbb{N}$ such that $\frac{\lambda_{n}^{j}}{\lambda_{n}^{i}}<C_{0}$ holds for all $n \in \mathbb{N}$ and $i, j=1, \ldots, m$.

- There is a constant $d_{0}>0$ such that $\left|x_{n}^{i}-x_{n}^{j}\right|>d_{0}$ for any $n \in \mathbb{N}$ and $i, j=1, \ldots, m$ with $i \neq j$.

- Let $b_{i}=\left(\lim _{n \rightarrow \infty} \frac{\lambda_{n}^{i}}{\lambda_{n}^{1}}\right)^{\frac{N-2 s}{2}}$ and $b_{0}=\lim _{n \rightarrow \infty}\left(\lambda_{n}^{1}\right)^{-(N-2 s)} \epsilon_{n}$. Then, the value

$$
\left(\left(b_{1}, \ldots, b_{m}\right),\left(x_{0}^{1}, \ldots, x_{0}^{m}\right)\right) \subset(0, \infty)^{m} \times \Omega^{m}
$$

is a critical point of the function $\Phi_{m}$ defined by

$$
\begin{aligned}
\Phi_{m}\left(b_{1}, \ldots, b_{m}, x_{1}, \ldots, x_{m}\right)=c_{1} & \left(\sum_{i=1}^{m} b_{i}^{2} H\left(x_{i}, x_{i}\right)-\sum_{i \neq k} b_{i} b_{k} G\left(x_{i}, x_{k}\right)\right) \\
& -c_{2} \log \left(b_{1} \ldots b_{m}\right) \cdot b_{0}
\end{aligned}
$$

where

$$
c_{1}=\int_{\mathbb{R}^{N}} w_{1,0}^{p} d x>0 \text { and } c_{2}=\left(\frac{N-2 s}{N}\right) \frac{\int_{\mathbb{R}^{N}} w_{1,0}^{p+1} d x}{\int_{\mathbb{R}^{N}} w_{1,0}^{p} d x}>0 .
$$

Here, $G: \Omega \times \Omega \rightarrow \mathbb{R}$ is Green's function of $(-\Delta)^{s}, H: \Omega \times \Omega \rightarrow \mathbb{R}$ is its regular part, and $w_{1,0}$ is the standard bubble on $\mathbb{R}^{N}$ given in (2.13). (See Sect. 2 for more details.)

Remark 1.2 As we mentioned, Eq. (1.1) may have a solution even for $\epsilon \leq 0$ if the topology of the domain $\Omega$ is not simple (say, its homology group over $\mathbb{Z} /(2 \mathbb{Z})$ is nontrivial). Hence, the first case (1) of Theorem 1.1 cannot be excluded for general domains.

If the blow-up points satisfy a certain nondegeneracy condition, then we can determine the blow-up rates in terms of an explicit power of $\epsilon^{-1}$ as the following theorem shows.

Theorem 1.3 Let $\left\{u_{n}\right\}_{n \in \mathbb{N}}$ be a sequence of solutions to (1.1) satisfying (2) of Theorem 1.1. Let us set an $m \times m$ symmetric matrix $M=\left(m_{i j}\right)_{1 \leq i, j \leq m}$ by

$$
m_{i j}= \begin{cases}H\left(x_{0}^{i}, x_{0}^{i}\right) & \text { if } i=j \\ -G\left(x_{0}^{i}, x_{0}^{j}\right) & \text { if } i \neq j .\end{cases}
$$

Then, it is nonnegative definite. If it is nondegenerate (i.e., positive definite), then for any $1 \leq i \leq m$, we have

$$
\lim _{n \rightarrow \infty} \log _{\epsilon_{n}} \lambda_{n}^{i}=\frac{1}{N-2 s}
$$

Recall that Eq. (1.1) has multi-bubble solutions as the following result indicates. 
Theorem A (Choi et al. [25]; Dávila et al. [29]) Assume $s \in(0,1)$ and $N>2 s$. Given arbitrary $m \in \mathbb{N}$, suppose that the function $\Phi_{m}$ in (1.6) with $b_{0}=\frac{N-2 s}{4 s}$ has a stable critical set $\Lambda_{m}$ such that

$$
\begin{gathered}
\Lambda_{m} \subset\left\{\left(\left(\lambda_{1}, \ldots, \lambda_{m}\right),\left(x_{1}, \ldots, x_{m}\right)\right) \in(0, \infty)^{m} \times \Omega^{m}:\right. \\
\left.x_{i} \neq x_{j} \text { if } i \neq j \text { and } i, j=1, \ldots, m\right\} .
\end{gathered}
$$

Then, there exists a point $\left(\left(\lambda_{0}^{1}, \ldots, \lambda_{0}^{m}\right),\left(x_{0}^{1}, \ldots, x_{0}^{m}\right)\right) \in \Lambda_{m}$ and a small number $\epsilon_{0}>0$ such that for $0<\epsilon<\epsilon_{0}$, there is a family of solutions $u_{\epsilon}$ of (1.1) which concentrate at each point $x_{0}^{1}, \ldots, x_{0}^{m-1}$ and $x_{0}^{m}$ as $\epsilon \rightarrow 0$ in the form (1.5), after extracting a subsequence if necessary.

The asymptotic behavior of solutions figured in Theorem 1.3 (2) corresponds exactly to the multi-peak solutions described in the above theorem. This reveals the accuracy and sharpness of our classification results. The question of finding a blow-up sequence of solutions not satisfying (1.8) is open even for the local case $s=1$.

Before introducing our strategy for the proof of the classification results, it is worth to remind that problem (1.1) is a nonlocal version of the Lane-Emden-Fowler equation

$$
\begin{cases}-\Delta u=u^{\frac{N+2}{N-2}-\epsilon} & \text { in } \Omega, \\ u>0 & \text { in } \Omega, \\ u=0 & \text { on } \partial \Omega .\end{cases}
$$

In [49], Rey constructed one-peak solutions to (1.9). Then, multi-peak solutions were found by Bahri et al. [5], Rey [51] and Musso and Pistoia [47] (for $N \geq 3$ ) by different ways. Furthermore, the classification of solutions was conducted in Han [38] and Rey [49] for one-peak case $(N \geq 3)$, and in Bahri et al. [5] and Rey [51] for general case $(N \geq 4$ and $N=3$, respectively).

Theorem B (Bahri et al. [5]; Rey [51]) Assume that $N \geq 3$ and $\left\{u_{n}\right\}_{n \in \mathbb{N}} \subset H_{0}^{1}(\Omega)$ is a sequence of solutions to (1.9) with $\epsilon=\epsilon_{n} \searrow 0$. Also, suppose that $\sup _{n \in \mathbb{N}}\left\|u_{n}\right\|_{H_{0}^{1}(\Omega)}<\infty$.

Passing to a subsequence, either $u_{n}$ strongly converges to a solution $u$ of (1.4) with $s=1$, or it has the asymptotic behavior (1.5) where $P w_{\lambda, \xi}$ is the projected bubble defined as

$$
-\Delta P w_{\lambda, \xi}=w_{\lambda, \xi}^{p} \text { in } \Omega \text { and } P w_{\lambda, \xi}=0 \text { on } \partial \Omega
$$

$\left(w_{\lambda, \xi}\right.$ is given in (2.13)). Moreover, all characteristics of the concentration points $\left\{x_{n}^{1}, \ldots, x_{n}^{m}\right\}$ and rates $\left\{\lambda_{n}^{1}, \ldots, \lambda_{n}^{m}\right\}$ in the statement of Theorem 1.1 remain to hold. If the nonnegative matrix $M$ defined in the statement of Theorem 1.3 is in fact positive, then (1.8) is valid.

In $[5,51]$, a certain decomposition of the space $H_{0}^{1}(\Omega)$ is crucially used (see Remark 1.4 (1) below), which produces large error in the lowest-dimension case $N=3$. In this reason, improved estimates had to be made additionally in [51]. Remarkably, as we will see later, our proof for theorems 1.1 and 1.3 provides a unified and neater approach to treat this local situation $s=1$. As a result, we have a new proof of Theorem B working for all dimensions $N \geq 3$ at the same time. See Sect. 6.2.

The framework of the proofs for our main theorems comprises of the following three steps:

Step 1. Concentration-compactness principle; 
Step 2. Pointwise bounds of $u_{n}$ obtained from a moving sphere argument and their applications;

Step 3. Two identities regarding Green's function and the Robin function coming from a type of Green's identity.

Let us briefly explain each step by assuming that the spectral fractional Laplacian is under consideration.

In Step 1, we recall the concentration-compactness principle for problem (1.1). This renowned principle is found by Struwe [59] for Eq. (1.9), and recently extended to problem (1.1) by Almaraz [2] for $s=\frac{1}{2}$, and by Fang and González [31], and Palatucci and Pisante [48] for all $0<s<1$ (in slightly different setting). It makes possible to decompose solutions $\left\{u_{n}\right\}_{n \in \mathbb{N}}$ of $(1.1)$ as

$$
u_{n}=v_{0}+\sum_{i=1}^{m} P w_{\lambda_{n}^{i}, x_{n}^{i}}+r_{n},
$$

where $v_{0}$ is the $\widetilde{H}^{s}(\Omega)$-weak limit of $\left\{u_{n}\right\}_{n \in \mathbb{N}}, P w_{\lambda_{n}^{i}, x_{n}^{i}} \in \widetilde{H}^{s}(\Omega)$ is the projected bubble and $r_{n}$ converges to zero in $\widetilde{H}^{s}(\Omega)$. See Lemma 2.2 for the complete description of $\lambda_{n}^{i}, x_{n}^{i}, v_{0}, P w_{\lambda, \xi}$ and $r_{n}$.

Now our task is reduced to getting further information on the sequence $\left\{u_{n}\right\}_{n \in \mathbb{N}}$ whose elements are expressed as (1.10), which is one of the main contributions of this paper. We immediately encounter a difficulty, because we do not know at this moment even whether two different concentration points $x_{n}^{i}$ and $x_{n}^{j}$ may collide or not. This technicality will be tackled in Step 2, where we attain a pointwise bound of $u_{n}$ near each concentration point by employing the moving sphere method toward the extended problem (2.4) of Eq. (1.1) (see Sect. 3). This allows us to deduce no coincidence of two different blow-up points and to obtain further valuable information on solutions such as the alternative between $v_{0}=0$ and $m=0$, and compatibility of blow-up rates of all peaks (see Sect. 4). This part is motivated by Schoen [55].

Given the pointwise bound and its consequences derived in Step 2, we show in Step 3 that the $L^{\infty}$-normalized sequence of the solutions $u_{n}$ converges to a combination of Green's functions. Then, inserting this information into a Green-type identity (5.3) will lead us to discover two identities (5.4) and (5.11) regarding on the limit of the blow-up profile $\left(\lambda_{n}^{1}, \ldots, \lambda_{n}^{m}, x_{n}^{1}, \ldots x_{n}^{m}\right)$, which will complete the proof of our main results. On passing to the limit, one needs to know a uniform $C^{2}$-estimate of the $s$-harmonic extensions of $\left\{u_{n}\right\}_{n \in \mathbb{N}}$. It is not a trivial issue since we are handling the nonlocal problem (1.1), or the associate degenerate local problem (2.4) with the weighted Neumann boundary condition. "Appendix 2 " is devoted to deduce the desired regularity results.

The above strategy extends Han's method [38] in a quite natural manner, while the argument in Bahri et al. [5] and Rey [51] can be regarded as further developments of Rey [49,50].

We conclude this section, presenting some additional remarks.

Remark 1.4 (1) The corresponding result to Step 3 for the local problem (1.9) was achieved in Bahri et al. [5] and Rey [51]. The argument in [5,51] requires one to estimate $\left\|r_{n}\right\|_{H_{0}^{1}(\Omega)}$ in terms of powers of $\epsilon_{n}$ and $\max _{1 \leq k \leq m} \lambda_{n}^{k}$. For this aim, the authors replaced $\sum_{i=1}^{m} P w_{\lambda_{n}^{i}, x_{n}^{i}}$ in the expansion (1.10) of $u_{n}$ with $\sum_{i=1}^{m} \alpha_{n}^{i} P w_{\lambda_{n}^{i}, x_{n}^{i}}$ (for some $\alpha_{n}^{i} \in \mathbb{R}$ ) and 
then perturbed the parameters $\left(\alpha_{n}^{i}, \lambda_{n}^{i}, x_{n}^{i}\right)$ so that $r_{n}$ satisfies the $H_{0}^{1}(\Omega)$-orthogonality

$$
\begin{aligned}
\left\langle r_{n}, P w_{\lambda_{n}^{i}, x_{n}^{i}}\right\rangle_{H_{0}^{1}(\Omega)} & =\left\langle r_{n}, \frac{\partial P w_{\lambda_{n}^{i}, x_{n}^{i}}}{\partial x_{j}}\right\rangle_{H_{0}^{1}(\Omega)} \\
& =\left\langle r_{n}, \frac{\partial P w_{\lambda_{n}^{i}, x_{n}^{i}}}{\partial \lambda}\right\rangle_{H_{0}^{1}(\Omega)}=0 \text { for } 1 \leq i \leq m, 1 \leq j \leq N,
\end{aligned}
$$

as in Bahri and Coron [4]. After that, they followed the argument of Rey [49,50] to get a sharp estimate $\left\|r_{n}\right\|_{H_{0}^{1}(\Omega)}$. Their argument is simplified in our proof in the point that we do not need the estimate of the remainder term $r_{n}$.

(2) An advantage of the argument in [5,51] is that it deals with the energy functional of (1.9) directly so that it suggests a way to compute the Morse index of the solutions. Recently, asymptotic behavior of the first $(N+2) m$-eigenvalues and eigenfunctions for the linearized equation of (1.9) was examined in [26,36]. They give the information on the Morse index as a particular corollary.

The rest of this paper is organized as follows. In Sect. 2, we review the extension problem for the spectral and restricted fractional Laplacians, Green's function, the Robin function and the projected bubbles. Moreover, we recall the concentration-compactness principle which brings with a decomposition result of blow-up solutions. Section 3 is devoted to the proof of a pointwise upper bound which makes use of a moving sphere argument. In Sect. 4, by using this estimate, we attain various refined information for the blow-up solutions, and in particular, show that suitably normalized blow-up solutions converge to combinations of Green's functions. In Sect. 5, we obtain essential information of the blow-up points and their blow-up rates by using a Green-type identity, which proves our main results. For the sake of brevity, we concentrate only on the spectral fractional Laplacian in Sects. 3-5. Instead, all necessary modifications to deal with the restricted fractional Laplacian or the classical (local) Laplacian are listed in Sect. 6. Finally, a decay estimate of the standard bubble $W_{1,0}$ (see Sect. 2.4) needed in Sect. 3 and elliptic regularity results necessary for Lemma 4.6 are derived in appendices 7 and 8, respectively.

\section{Notations.}

- The letter $z$ represents a variable in the half-space $\mathbb{R}_{+}^{N+1}=\mathbb{R}^{N} \times(0, \infty)$. Also, it is written as $z=(x, t)=\left(x_{1}, \ldots, x_{N}, x_{N+1}\right)$ with $x=\left(x_{1}, \ldots, x_{N}\right) \in \mathbb{R}^{N}$ and $t=x_{N+1}>0$.

- For any fixed smooth open bounded domain $\Omega \subset \mathbb{R}^{N}$, let $\mathcal{C}:=\Omega \times(0, \infty) \subset \mathbb{R}_{+}^{N+1}$ be the associated cylinder of $\Omega$ and $\partial_{L} \mathcal{C}:=\partial \Omega \times(0, \infty)$ its lateral boundary. Set also $\mathcal{C}^{\prime}:=\Omega \times[0, \infty)$.

- For fixed $N \in \mathbb{N}$ and $s \in(0,1)$ such that $N>2 s$, the weighted Sobolev space $D^{1,2}\left(\mathbb{R}_{+}^{N+1} ; t^{1-2 s}\right)$ is defined as the completion of the space $C_{c}^{\infty}\left(\overline{\mathbb{R}_{+}^{N+1}}\right)$ with respect to the norm

$$
\|U\|_{D^{1,2}\left(\mathbb{R}_{+}^{N+1} ; t^{1-2 s}\right)}:=\left(\int_{\mathbb{R}_{+}^{N+1}} t^{1-2 s}|\nabla U(z)|^{2} d z\right)^{1 / 2} \text { for } U \in C_{c}^{\infty} \overline{\left(\mathbb{R}_{+}^{N+1}\right) .}
$$

Moreover, for any given cylinder $\mathcal{C}=\Omega \times(0, \infty)$ (where $\Omega$ is a smooth open bounded domain), the space $H_{0}^{1,2}\left(\mathcal{C} ; t^{1-2 s}\right)$ is the completion of $C_{c}^{\infty}(\mathcal{C} \cup(\Omega \times\{0\}))$ with respect to the above norm.

- We will denote by $p$ the critical exponent $\frac{N+2 s}{N-2 s}$. 
- Let $B_{+}^{N+1}((x, 0), r)$ be the half-ball in $\mathbb{R}_{+}^{N+1}$ of radius $r$ centered at $(x, 0) \in \mathbb{R}^{N} \times\{0\}$. Moreover, we set $\partial_{I} B_{+}^{N+1}(0, r)=\partial B_{+}^{N+1}(0, r) \cap \mathbb{R}^{N+1}$.

- $d S$ stands for the surface measure. Also, a subscript attached to $d S$ (such as $d S_{x}$ or $d S_{z}$ ) denotes the variable of the surface.

- For an arbitrary domain $D \subset \mathbb{R}^{n}$, the map $v=\left(v_{1}, \ldots, v_{n}\right): \partial D \rightarrow \mathbb{R}^{n}$ denotes the outward unit normal vector on $\partial D$.

- Suppose that $D$ is a domain and $T \subset \partial D$. If $f$ is a function on $D$, then the trace of $f$ on $T$ is denoted by $\left.\operatorname{tr}\right|_{T} f$ whenever it is well defined.

- $\left|S^{N-1}\right|=2 \pi^{N / 2} / \Gamma(N / 2)$ denotes the Lebesgue measure of $(N-1)$-dimensional unit sphere $S^{N-1}$.

- The following positive constants will appear in (2.1), (2.2), (2.3), (2.8), (2.13) and (2.14):

$$
\begin{gathered}
c_{N, s}:=\frac{2^{2 s} s \Gamma\left(\frac{N+2 s}{2}\right)}{\pi^{\frac{N}{2}} \Gamma(1-s)}, \quad \kappa_{s}:=\frac{\Gamma(s)}{2^{1-2 s} \Gamma(1-s)}, \quad p_{N, s}:=\frac{\Gamma\left(\frac{N+2 s}{2}\right)}{\pi^{\frac{N}{2}} \Gamma(s)}, \\
\gamma_{N, s}:=\frac{1}{\left|S^{N-1}\right|} \cdot \frac{2^{1-2 s} \Gamma\left(\frac{N-2 s}{2}\right)}{\Gamma\left(\frac{N}{2}\right) \Gamma(s)}, \\
\alpha_{N, s}:=2^{\frac{N-2 s}{2}}\left(\frac{\Gamma\left(\frac{N+2 s}{2}\right)}{\Gamma\left(\frac{N-2 s}{2}\right)}\right)^{\frac{N-2 s}{4 s}} \text { and } \mathcal{S}_{N, s}:=2^{-s} \pi^{-\frac{s}{2}}\left(\frac{\Gamma\left(\frac{N-2 s}{2}\right)}{\Gamma\left(\frac{N+2 s}{2}\right)}\right)^{\frac{1}{2}}\left(\frac{\Gamma(N)}{\Gamma\left(\frac{N}{2}\right)}\right)^{\frac{s}{N}} .
\end{gathered}
$$

$-C>0$ is a generic value that may vary from line to line.

\section{Preliminaries on fractional Laplacians}

In this section, we review some preliminary notions and results which will be needed throughout the proofs of the main theorems.

\subsection{Definition of Sobolev spaces and fractional Laplacians}

For any smooth open bounded domain $\Omega$, let $\left\{\lambda_{k}, \phi_{k}\right\}_{k=1}^{\infty}$ be a sequence of the eigenvalues and the corresponding $L^{2}(\Omega)$-normalized eigenvectors of the Dirichlet Laplacian $-\Delta$ in $\Omega$,

$$
\left\{\begin{array}{l}
-\Delta \phi_{k}=\lambda_{k} \phi_{k} \text { in } \Omega \text { and } \phi_{k}=0 \text { on } \partial \Omega, \\
\left\|\phi_{k}\right\|_{L^{2}(\Omega)}=1
\end{array}\right.
$$

where $0<\lambda_{1}<\lambda_{2} \leq \lambda_{3} \leq \cdots$. Then the spectral Laplacian is defined in terms of the quadratic form

$$
Q_{\mathrm{spec}}^{s}(u):=\sum_{k=1}^{\infty} \lambda_{k}^{s}\left(\int_{\Omega} u \phi_{k} d x\right)^{2} \text { for } u \in \mathcal{V}^{s}(\Omega)
$$

where the domain $\mathcal{V}^{s}(\Omega)$ of the form $Q_{\mathrm{spec}}^{s}$ is

$$
\mathcal{V}^{s}(\Omega):=\left\{u \in L^{2}(\Omega): Q_{\mathrm{spec}}^{s}(u)<\infty\right\} .
$$

By [46, Lemma 1] and [17, Proposition 2.1], it is known that

$$
\mathcal{V}^{s}(\Omega)=\widetilde{H}^{s}(\Omega)=\left\{u=\left.\operatorname{tr}\right|_{\Omega \times\{0\}} U: U \in H_{0}^{1,2}\left(\mathcal{C} ; t^{1-2 s}\right)\right\}
$$


where $\widetilde{H}^{s}(\Omega)$ is the function space defined in (1.3).

On the other hand, for any $s \in(0,1)$ and $u \in H^{s}\left(\mathbb{R}^{N}\right)$, we are capable of defining the fractional Laplacian by using the integral representation

$$
(-\Delta)^{s} u(x)=c_{N, s} \text { P.V. } \int_{\mathbb{R}^{N}} \frac{u(x)-u(y)}{|x-y|^{N+2 s}} d y .
$$

Here, the exact value of $c_{N, s}>0$ (as well as other constants such as $\kappa_{s}$ or $p_{N, s}$ below) can be found at the last part of the previous section. If this operator is restricted to functions in $\widetilde{H}^{s}(\Omega)$, then it is called the restricted fractional Laplacian.

To compare two different fractional Laplacians, the reader is advised to check the papers $[9,46,56]$. Given either the spectral or restricted fractional Laplacian, we set

$$
\|u\|_{\widetilde{H}^{s}(\Omega)}=\left(\int_{\Omega}\left|(-\Delta)^{s / 2} u\right|^{2} d x\right)^{\frac{1}{2}}
$$

for any $u \in \widetilde{H}^{s}(\Omega)$.

Remark 2.1 A Moser iteration argument combined with the use of the Caffarelli-Silvestre extension [15] shows that any finite energy solution $u$ of (1.1) is bounded (see, for example, [25]), and elliptic regularity results guarantee that $u$ is continuous up to the boundary (refer to [16,37] for the spectral case and [52] for the restricted case). Hence, it makes sense to say that a finite energy solution $u$ to (1.1) has zero boundary values.

\subsection{Localization of fractional Laplacians}

Let $\Omega$ be a smooth open bounded domain. For a fixed function $u \in \mathcal{V}^{s}(\Omega)=\widetilde{H}^{s}(\Omega)$ (or $H^{s}\left(\mathbb{R}^{N}\right)$ ), let us set $U \in H_{0}^{1,2}\left(\mathcal{C} ; t^{1-2 s}\right)$ (or $D^{1,2}\left(\mathbb{R}_{+}^{N+1} ; t^{1-2 s}\right)$, respectively) to be the $s$-harmonic extension of $u$, namely, a unique solution of the equation

$$
\begin{cases}\operatorname{div}\left(t^{1-2 s} \nabla U\right)=0 & \text { in } \mathcal{C} \quad\left(\text { or } \mathbb{R}_{+}^{N+1}\right), \\ U=0 & \text { on } \partial_{L} \mathcal{C} \quad\left(\text { or } \partial_{L} \mathbb{R}_{+}^{N+1}=\emptyset\right), \\ U(\cdot, 0)=u & \text { on } \Omega\left(\text { or } \mathbb{R}^{N}\right) .\end{cases}
$$

Then, by the celebrated results of Caffarelli and Silvestre [15] (for the Euclidean space $\mathbb{R}^{N}$ ) and Cabré-Tan [14] (for bounded domains $\Omega$, see also [17,58,61]), it holds that

$$
(-\Delta)^{s} u(x)=\partial_{\nu}^{s} U(x):=-\kappa_{s} \lim _{t \rightarrow 0+} t^{1-2 s} \frac{\partial U}{\partial t}(x, t) \text { for } x \in \Omega\left(\text { or } \mathbb{R}^{N}\right) .
$$

Moreover, if $u \in H^{s}\left(\mathbb{R}^{N}\right)$, then the Poisson representation formula gives that

$$
U(x, t)=p_{N, s} \int_{\mathbb{R}^{N}} \frac{t^{2 s}}{\left(|x-y|^{2}+t^{2}\right)^{\frac{N+2 s}{2}}} u(y) d y
$$

while for $u \in \mathcal{V}^{s}(\Omega)$ it is possible to describe $U$ in terms of a series related to the Bessel functions (refer to [17]). 
As a result, if the spectral fractional Laplacian is concerned, then the $s$-harmonic extension $U_{\epsilon} \in H_{0}^{1,2}\left(\mathcal{C} ; t^{1-2 s}\right)$ of a solution $u_{\epsilon} \in \mathcal{V}^{s}(\Omega)$ to problem (1.1) satisfies

$$
\begin{cases}\operatorname{div}\left(t^{1-2 s} \nabla U_{\epsilon}\right)=0 & \text { in } \mathcal{C}, \\ U_{\epsilon}=0 & \text { on } \partial_{L} \mathcal{C}, \\ U_{\epsilon}=u_{\epsilon} & \text { on } \Omega \times\{0\}, \\ \partial_{\nu}^{s} U_{\epsilon}=u_{\epsilon}^{p-\epsilon} & \text { on } \Omega \times\{0\} .\end{cases}
$$

In light of the Sobolev inequality (refer to [17, Sections 1-2] and (2.14) below), we see

$$
\left\|U_{\epsilon}\right\|_{H_{0}^{1,2}\left(\mathcal{C} ; t^{1-2 s}\right)}^{2}=\left\|u_{\epsilon}\right\|_{L^{p+1-\epsilon}(\Omega)}^{p+1-\epsilon} \leq C\left\|u_{\epsilon}\right\|_{\widetilde{H}^{s}(\Omega)}^{p+1-\epsilon} .
$$

Therefore, if we have $\sup _{\epsilon>0}\left\|u_{\epsilon}\right\|_{\widetilde{H}^{s}(\Omega)}<+\infty$, then $\sup _{\epsilon>0}\left\|U_{\epsilon}\right\|_{H_{0}^{1,2}\left(\mathcal{C} ; t^{1-2 s}\right)}<+\infty$. Moreover, by the strong maximum principle ([12, Corollary 4.12] or [30, Lemma 2.7]), it holds that $U_{\epsilon}>0$ in $\mathcal{C}$.

A similar (and in fact simpler) formulation is available when the restricted fractional Laplacian is studied. In this case, the equation we have to consider is

$$
\begin{cases}\operatorname{div}\left(t^{1-2 s} \nabla U_{\epsilon}\right)=0 & \text { in } \mathbb{R}_{+}^{N+1}, \\ U_{\epsilon}=0 & \text { on }\left(\mathbb{R}^{N} \backslash \Omega\right) \times\{0\}, \\ U_{\epsilon}=u_{\epsilon} & \text { on } \Omega \times\{0\}, \\ \partial_{v}^{s} U_{\epsilon}=u_{\epsilon}^{p-\epsilon} & \text { on } \Omega \times\{0\} .\end{cases}
$$

\subsection{Green's functions of fractional Laplacians}

In this subsection, we review Green's functions.

We consider first the case when the fractional Laplacian is defined in terms of the spectra of the Laplacian. We refer to [25] for more details.

Let $G$ be Green's function of the spectral fractional Laplacian $(-\Delta)^{s}$ on a smooth open bounded domain $\Omega$ with the zero Dirichlet boundary condition. Then, it can be regarded as the trace of Green's function $G_{\mathcal{C}}=G_{\mathcal{C}}(z, x)(z \in \mathcal{C}, x \in \Omega)$ for the Dirichlet-Neumann problem on the extended domain $\mathcal{C}$ which satisfies

$$
\begin{cases}\operatorname{div}\left(t^{1-2 s} \nabla G_{\mathcal{C}}(\cdot, x)\right)=0 & \text { in } \mathcal{C}, \\ G_{\mathcal{C}}(\cdot, x)=0 & \text { on } \partial_{L} \mathcal{C}, \\ \partial_{v}^{s} G_{\mathcal{C}}(\cdot, x)=\delta_{x} & \text { on } \Omega \times\{0\}\end{cases}
$$

where $\delta_{x}$ is the Dirac delta function on $\mathbb{R}^{n}$ with center at $x \in \Omega$.

Green's function $G_{\mathcal{C}}$ on the half-cylinder $\mathcal{C}$ can be decomposed into the singular and regular parts. The singular part is given by Green's function

$$
G_{\mathbb{R}_{+}^{N+1}}((x, t), y):=\frac{\gamma_{N, s}}{|(x-y, t)|^{N-2 s}}
$$

on the half-space $\mathbb{R}_{+}^{N+1}$ satisfying

$$
\begin{cases}\operatorname{div}\left(t^{1-2 s} \nabla_{(x, t)} G_{\mathbb{R}_{+}^{N+1}}((x, t), y)\right)=0 & \text { in } \mathbb{R}_{+}^{N+1}, \\ \partial_{v}^{s} G_{\mathbb{R}_{+}^{N+1}}((x, 0), y)=\delta_{y}(x) & \text { on } \mathbb{R}^{N}=\partial \mathbb{R}_{+}^{N+1}\end{cases}
$$


for each $y \in \mathbb{R}^{N}$. The regular part is given as the function $H_{\mathcal{C}}: \mathcal{C} \rightarrow \mathbb{R}$ which solves

$$
\begin{cases}\operatorname{div}\left(t^{1-2 s} \nabla_{(x, t)} H_{\mathcal{C}}((x, t), y)\right)=0 & \text { in } \mathcal{C}, \\ H_{\mathcal{C}}((x, t), y)=\frac{\gamma_{N, s}}{|(x-y, t)|^{N-2 s}} & \text { on } \partial_{L} \mathcal{C}, \\ \partial_{v}^{s} H_{\mathcal{C}}((x, 0), y)=0 & \text { on } \Omega \times\{0\}\end{cases}
$$

for any $y \in \Omega$. Its existence can be verified in a variational method (see Lemma 2.2 in [25]). We then have

$$
G_{\mathcal{C}}((x, t), y)=G_{\mathbb{R}_{+}^{N+1}}((x, t), y)-H_{\mathcal{C}}((x, t), y) .
$$

Now, letting $H(x, y)=H_{\mathcal{C}}((x, 0), y)$, we can decompose $G(x, y)=G_{\mathcal{C}}((x, 0), y)$ as follows.

$$
G(x, y)=\frac{\gamma_{N, s}}{|x-y|^{N-2 s}}-H(x, y) .
$$

Let us recall some regularity properties of the function $H_{\mathcal{C}}$. For any index $\alpha \in(\mathbb{N} \cup\{0\})^{N}$, the partial derivatives $\partial_{x}^{\alpha} H_{\mathcal{C}}$ of $H_{\mathcal{C}}$ in the $x$-variable always exist (see Lemma 8.1 and Sect. 2 of [25]). In addition, it follows from (2.10) that

$$
\begin{cases}\operatorname{div}\left(t^{1-2 s} \nabla_{(x, t)} \partial_{x}^{\alpha} H_{\mathcal{C}}((x, t), y)\right)=0 & \text { in } \mathcal{C}, \\ \partial_{v}^{s} \partial_{x}^{\alpha} H_{\mathcal{C}}((x, 0), y)=0 & \text { on } \Omega \times\{0\} .\end{cases}
$$

Therefore, by applying [12, Lemma 4.5] to each $\partial_{x}^{\alpha} H_{\mathcal{C}}$, we see that there is a constant $C=C(\alpha, r, \xi)>0$ such that

$$
\left|\partial_{x}^{\alpha} H_{\mathcal{C}}((x, t), y)\right| \leq C
$$

and

$$
\left|t^{1-2 s} \partial_{t} \partial_{x}^{\alpha} H_{\mathcal{C}}((x, t), y)\right| \leq C
$$

for all $(x, t) \in B_{+}^{N+1}((\xi, 0), r)$ provided that $\xi \in \Omega$ and $r>0$ satisfy the condition $r<\operatorname{dist}(\xi, \partial \Omega)$.

When the restricted fractional Laplacian is dealt with, we observe that the above discussion is still valid once we let $\mathcal{C}=\mathbb{R}_{+}^{N+1}$ and substitute the boundary conditions in (2.7) and (2.10) with

$$
\begin{aligned}
G_{\mathcal{C}}(\cdot, x) & =0 \text { on } \partial_{B} \mathcal{C} \text { and } \\
H_{\mathcal{C}}((x, t), y) & =\frac{\gamma_{N, s}}{|(x-y, t)|^{N-2 s}} \quad \text { on } \partial_{B} \mathcal{C}
\end{aligned}
$$

respectively, where $\partial_{B} \mathcal{C}:=\left(\mathbb{R}^{N} \backslash \Omega\right) \times\{0\}$. (The function $G_{\mathcal{C}}$ in this paragraph should not be confused with the fundamental solution $G_{\mathbb{R}_{+}^{N+1}}$ in (2.8).)

\subsection{Sharp Sobolev and trace inequalities}

Given any $\lambda>0$ and $\xi \in \mathbb{R}^{N}$, let $w_{\lambda, \xi}$ be the bubble defined by

$$
w_{\lambda, \xi}(x)=\alpha_{N, s}\left(\frac{\lambda}{\lambda^{2}+|x-\xi|^{2}}\right)^{\frac{N-2 s}{2}} \text { for } x \in \mathbb{R}^{N} .
$$


Then, it is true that

$$
\left(\int_{\mathbb{R}^{N}}|u|^{p+1} \mathrm{~d} x\right)^{\frac{1}{p+1}} \leq \mathcal{S}_{n, s}\left(\int_{\mathbb{R}^{N}}\left|(-\Delta)^{s / 2} u\right|^{2} \mathrm{~d} x\right)^{\frac{1}{2}} \quad \text { for all } u \in H^{s}\left(\mathbb{R}^{N}\right),
$$

and the equality holds if and only if $u(x)=c w_{\lambda, \xi}(x)$ for any $c>0, \lambda>0$ and $\xi \in \mathbb{R}^{N}$ (refer to $[18,33,45]) .{ }^{1}$ Furthermore, it was shown in $[20,42,44]$ that if a suitable decay assumption is imposed, then $\left\{w_{\lambda, \xi}: \lambda>0, \xi \in \mathbb{R}^{N}\right\}$ is the set of all solutions for the problem

$$
(-\Delta)^{s} u=u^{p}, \quad u>0 \text { in } \mathbb{R}^{N} \text { and } \lim _{|x| \rightarrow \infty} u(x)=0 .
$$

Denote also the $s$-harmonic extension of $w_{\lambda, \xi}$ by $W_{\lambda, \xi} \in D^{1,2}\left(\mathbb{R}_{+}^{N+1} ; t^{1-2 s}\right)$ so that $W_{\lambda, \xi}$ solves

$$
\begin{cases}\operatorname{div}\left(t^{1-2 s} W_{\lambda, \xi}(x, t)\right)=0 & \text { in } \mathbb{R}_{+}^{N+1} \\ W_{\lambda, \xi}(x, 0)=w_{\lambda, \xi}(x) & \text { on } \mathbb{R}^{N}\end{cases}
$$

It follows that for the Sobolev trace inequality

$$
\left(\int_{\mathbb{R}^{N}}|U(x, 0)|^{p+1} \mathrm{~d} x\right)^{\frac{1}{p+1}} \leq \sqrt{\kappa_{s}} \mathcal{S}_{n, s}\left(\int_{0}^{\infty} \int_{\mathbb{R}^{N}} t^{1-2 s}|\nabla U(x, t)|^{2} \mathrm{~d} x \mathrm{~d} t\right)^{\frac{1}{2}},
$$

the two sides are equal if and only if $U(x, t)=c W_{\lambda, \xi}(x, t)$ for any $c>0, \lambda>0$ and $\xi \in \mathbb{R}^{N}$.

\subsection{Concentration-compactness principle}

Firstly, we treat the spectral fractional Laplacian case. Let $P W_{\lambda, \xi}$ stand for the projection of the bubble $W_{\lambda, \xi}$ into $H_{0}^{1,2}\left(\mathcal{C} ; t^{1-2 s}\right)$, that is, the solution of

$$
\begin{cases}\operatorname{div}\left(t^{1-2 s} \nabla P W_{\lambda, \xi}\right)=0 & \text { in } \mathcal{C}, \\ P W_{\lambda, \xi}=0 & \text { on } \partial_{L} \mathcal{C}, \\ \partial_{\nu}^{s} P W_{\lambda, \xi}=\partial_{\nu}^{s} W_{\lambda, \xi}=W_{\lambda, \xi}^{p} & \text { on } \Omega \times\{0\},\end{cases}
$$

and $P w_{\lambda, \xi}=\left.\operatorname{tr}\right|_{\Omega \times\{0\}} P W_{\lambda, \xi}$. By the maximum principle [25, Lemma 2.1], we have $0 \leq$ $P W_{\lambda, \xi} \leq W_{\lambda, \xi}$ in $\mathcal{C}$. Also [25, Lemma C.1] says that

$$
P W_{\lambda, \xi}(z)=W_{\lambda, \xi}(z)-c_{1} \lambda^{\frac{N-2 s}{2}} H(z, \xi)+o\left(\lambda^{\frac{N-2 s}{2}}\right)
$$

uniformly for $z \in \mathcal{C}$ where $c_{1}>0$ is the number appeared in (1.6).

The following result is a fractional version of Struwe [59].

Lemma 2.2 Let $\left\{U_{n}\right\}_{n \in \mathbb{N}}$ be a sequence of solutions to (2.4) with $\epsilon=\epsilon_{n} \searrow 0$ which satisfies the norm condition $\sup _{n \in \mathbb{N}}\left\|U_{n}\right\|_{H_{0}^{1,2}\left(\mathcal{C} ; t^{1-2 s}\right)}<\infty$. Then, there exist an integer $m \in \mathbb{N} \cup\{0\}$ and a sequence $\left\{\left(\lambda_{n}^{i}, x_{n}^{i}\right)\right\}_{n \in \mathbb{N}} \subset(0, \infty) \times \Omega$ of positive numbers and points for each $i=1, \ldots$, m such that

$$
R_{n}:=U_{n}-\left(V_{0}+\sum_{i=1}^{m} P W_{\lambda_{n}^{i}, x_{n}^{i}}\right) \rightarrow 0 \text { in } H_{0}^{1,2}\left(\mathcal{C} ; t^{1-2 s}\right) \text { as } n \rightarrow \infty
$$

${ }_{1}^{1}$ The constant $\mathcal{S}_{n, s}$ is still the best constant even if we restrict the class $H^{s}\left(\mathbb{R}^{N}\right)$ to the subspace $\widetilde{H}^{s}(\Omega)$. See [46, Theorem 4]. 
(up to a subsequence) where $V_{0}$ is the weak limit of $U_{n}$ in $H_{0}^{1,2}\left(\mathcal{C} ; t^{1-2 s}\right)$, which satisfies

$$
\begin{cases}\operatorname{div}\left(t^{1-2 s} \nabla V_{0}\right)=0 & \text { in } \mathcal{C}, \\ V_{0}=0 & \text { on } \partial_{L} \mathcal{C}, \\ \partial_{v}^{s} V_{0}=V_{0}^{\frac{N+2 s}{N-2 s}} & \text { on } \Omega \times\{0\} .\end{cases}
$$

In addition, it holds that

$$
\frac{1}{\lambda_{n}^{i}} \operatorname{dist}\left(x_{n}^{i}, \partial \Omega\right) \rightarrow \infty \text { and } \frac{\lambda_{n}^{i}}{\lambda_{n}^{j}}+\frac{\lambda_{n}^{j}}{\lambda_{n}^{i}}+\frac{1}{\lambda_{n}^{i} \lambda_{n}^{j}}\left|x_{n}^{i}-x_{n}^{j}\right|^{2} \rightarrow \infty \text { as } n \rightarrow \infty
$$

for all $1 \leq i \neq j \leq m$.

Proof See [2] and [31] where an analogous conclusion is deduced in the setting of asymptotically hyperbolic manifolds. Since their approach still works for our case, we omit the proof.

Let $v_{0}=\left.\operatorname{tr}\right|_{\Omega \times\{0\}} V_{0}$ and $r_{n}=\left.\operatorname{tr}\right|_{\Omega \times\{0\}} R_{n}$.

Extracting a subsequence of $\left\{U_{n}\right\}_{n \in \mathbb{N}}$ and reordering the indices if necessary, we may assume that

$$
\lambda_{n}^{1} \leq \lambda_{n}^{2} \leq \cdots \leq \lambda_{n}^{m} \text { for all } n \in \mathbb{N} \text { and } x_{n}^{i} \rightarrow x_{0}^{i} \in \bar{\Omega} \text { as } n \rightarrow \infty .
$$

Using the Kelvin transform and the moving plane argument, Choi [23, Lemma 4.1] proved that $\left\{U_{n}\right\}_{n \in \mathbb{N}}$ are uniformly bounded near the boundary $\partial \Omega \times[0, \infty)$. That is, there exist constants $\delta>0$ and $C>0$ such that

$$
\sup _{n \in \mathbb{N}} \sup _{\{(x, t) \in \mathcal{C}: \operatorname{dist}(x, \partial \Omega)<\delta\}}\left|U_{n}(x, t)\right| \leq C .
$$

Hence,

$$
\operatorname{dist}\left(x_{0}^{i}, \partial \Omega\right) \geq \delta \text { for } i=1, \ldots, m .
$$

For the restricted fractional Laplacian, we define $P W_{\lambda, \xi}$ by (2.17) whose second line is replaced with $P W_{\lambda, \xi}=0$ in $\mathbb{R}^{N} \backslash \Omega$. Then, it is not hard to draw analogous results to Lemma 2.2 (cf. [48]) and (2.18). Besides, one can check that (2.23) still holds as follows: If the domain $\Omega$ is strictly convex, we apply the moving plane method with the maximum principle for small domains (given in [53, Lemma 5.1]), getting

$$
\sup _{n \in \mathbb{N}} \sup _{\operatorname{dist}(x, \partial \Omega)<\delta}\left|u_{n}(x)\right| \leq C .
$$

In the case that $\Omega$ does not have the convexity assumption, we first use the conformal invariance of Eq. (1.1) (refer to [52, Proposition A.1]) and then employ the moving plane method to obtain (2.24). Now combining (2.19) and (2.24) gives (2.23) at once. See [38, Section 2] to recall the argument used for the local case $s=1$.

In the next two sections, further information on blow-up rates $\left\{\lambda_{n}^{i}\right\}_{i=1}^{m}$ and points $\left\{x_{n}^{i}\right\}_{i=1}^{m}$ in the decomposition (2.19) will be examined. In what follows, we simply denote $w_{1,0}$ and $W_{1,0}$ by $w$ and $W$, respectively. Since $W=W(x, t)$ is radially symmetric in the $x$-variable, we will often write $W(x, t)=W(\rho, t)$ where $\rho=|x|$. In addition, the operator $(-\Delta)^{s}$ is understood as the spectral fractional Laplacian in Sects. 3, 4 and 5. Consideration on the restricted fractional Laplacian is postponed to Sect. 6. 


\section{Moving sphere argument and pointwise upper bound}

The aim of this section is to obtain a sharp pointwise upper bound of solutions $U_{\epsilon}$ to (2.4). To this end, we will employ the method of moving spheres (refer to $[21,43,55]$ ).

Proposition 3.1 Let $r_{0}>0$ be any fixed small number. Assume that $\left\{M_{\epsilon}\right\}_{\epsilon>0}$ is a family of positive numbers such that $\lim _{\epsilon \rightarrow \infty} M_{\epsilon}=\infty$ and $\lim _{\epsilon \rightarrow \infty} M_{\epsilon}^{\epsilon}=1$. If a family $\left\{V_{\epsilon}\right\}_{\epsilon>0}$ of positive functions satisfies

$$
\begin{cases}\operatorname{div}\left(t^{1-2 s} \nabla V_{\epsilon}\right)=0 & \operatorname{in} B^{N}\left(0, r_{0} M_{\epsilon}^{\frac{2}{N-2 s}}\right) \times(0, \infty), \\ \partial_{v}^{s} V_{\epsilon}=V_{\epsilon}^{p-\epsilon} & \text { on } B^{N}\left(0, r_{0} M_{\epsilon}^{\frac{2}{N-2 s}}\right), \\ \left\|V_{\epsilon}\right\|_{L^{\infty}\left(B_{+}^{N+1}\left(0, r_{0} M_{\epsilon}^{\frac{2}{N-2 s}}\right)\right)} \leq c & \end{cases}
$$

for some $c>0$, and

$$
V_{\epsilon} \rightarrow W \text { weakly in } D^{1,2}\left(\mathbb{R}_{+}^{N+1} ; t^{1-2 s}\right) \text { as } \epsilon \rightarrow 0,
$$

then there are constants $C>0$ and $0<\delta_{0}<r_{0}$ independent of $\epsilon>0$ such that

$$
V_{\epsilon}(z) \leq C W(z) \text { for all } z \in B_{+}^{N+1}\left(0, \delta_{0} M_{\epsilon}^{\frac{2}{N-2 s}}\right) .
$$

For the proof of the above proposition, we make some remarks.

Remark 3.2 (1) By (3.1), (3.2) and the Hölder regularity due to Cabre-Sire [12], if a constant $\zeta_{1}>0$ and a compact set $K \subset \overline{\mathbb{R}_{+}^{N+1}}$ are given, then there exist $\epsilon_{1}>0$ small and $\alpha \in(0,1)$ such that

$$
\left\|V_{\epsilon}-W\right\|_{C^{\alpha}(K)} \leq \zeta_{1} \text { for } \epsilon \in\left(0, \epsilon_{1}\right) .
$$

(2) For any function $F$ in $\mathbb{R}_{+}^{N+1}$, let $F^{\lambda}$ be its Kelvin transform of defined as

$$
F(z)=\left(\frac{\lambda}{|z|}\right)^{N-2 s} F\left(z^{\lambda}\right) \text { where } z^{\lambda}:=\frac{\lambda^{2} z}{|z|^{2}} \in \mathbb{R}_{+}^{N+1} .
$$

If we write $D_{\epsilon}^{\lambda}=V_{\epsilon}-V_{\epsilon}^{\lambda}$, then it holds that

$$
\begin{aligned}
\partial_{\nu}^{s} D_{\epsilon}^{\lambda} & =V_{\epsilon}^{p-\epsilon}-\left(\frac{\lambda}{|x|}\right)^{(N-2 s) \epsilon}\left(V_{\epsilon}^{\lambda}\right)^{p-\epsilon} \geq V_{\epsilon}^{p-\epsilon}-\left(V_{\epsilon}^{\lambda}\right)^{p-\epsilon} \\
& =\xi_{\epsilon}(x) D_{\epsilon}^{\lambda} \text { for }|x| \geq \lambda \text { and } t=0
\end{aligned}
$$

where

$$
\xi_{\epsilon}(x)= \begin{cases}\frac{V_{\epsilon}^{p-\epsilon}-\left(V_{\epsilon}^{\lambda}\right)^{p-\epsilon}}{V_{\epsilon}-V_{\epsilon}^{\lambda}}(x, 0) & \text { if } V_{\epsilon}(x, 0) \neq V_{\epsilon}^{\lambda}(x, 0), \\ (p-\epsilon) V_{\epsilon}^{p-1-\epsilon}(x, 0) & \text { if } V_{\epsilon}(x, 0)=V_{\epsilon}^{\lambda}(x, 0) .\end{cases}
$$

(3) For each $R>0$, let us introduce Green's function $G^{R}$ of the spectral fractional Laplacian $(-\Delta)^{S}$ in $\Omega=B^{N}(0, R)$ with zero Dirichlet boundary condition and Green's function $G_{\mathcal{C}}^{R}$ of Eq. (2.7) in the cylinder $\mathcal{C}=B^{N}(0, R) \times(0, \infty)$. By the scaling invariance, we have

$$
G^{R}(x, y)=\frac{1}{R^{N-2 s}} G^{1}\left(\frac{x}{R}, \frac{y}{R}\right) \quad \text { for } x, y \in B^{N}(0, R)
$$


and

$$
G_{\mathcal{C}}^{R}((x, t), y)=\frac{1}{R^{N-2 s}} G_{\mathcal{C}}^{1}\left(\left(\frac{x}{R}, \frac{t}{R}\right), \frac{y}{R}\right) \text { for } x, y \in B^{N}(0, R) \text { and } t>0 .
$$

Thus, we can decompose Green's function in $B^{N}(0, R)$ into its singular part and regular part as follows:

$$
\begin{aligned}
G_{\mathcal{C}}^{R}((x, t), y)= & \frac{\gamma_{N, s}}{|(x-y, t)|^{N-2 s}}-\frac{1}{R^{N-2 s}} H_{\mathcal{C}}^{1}\left(\left(\frac{x}{R}, \frac{t}{R}\right), \frac{y}{R}\right) \text { for } x, y \\
& \in B^{N}(0, R), t>0 .
\end{aligned}
$$

The precise value of the normalizing constant $\gamma_{n}$ is given in Notations.

As a preliminary step, we prove the minimum of $V_{\epsilon}$ on any half-sphere $\left\{z \in \mathbb{R}_{+}^{N+1}:|z|=\right.$ $r$ \} is controlled by the value $W(r, 0)$ whenever $r$ is at most of order $M_{\epsilon}^{\frac{2}{N-2 s}}$ and $\epsilon>0$ is small enough.

Lemma 3.3 Let $\left\{V_{\epsilon}\right\}_{\epsilon>0}$ be the family in the statement of Proposition 3.1. Then, for any $\zeta_{2}>0$, there exist small constants $\delta_{1} \in\left(0, r_{0}\right)$ and $\epsilon_{2}>0$ such that

$$
\min _{\left\{z \in \mathbb{R}_{+}^{N+1}:|z|=r\right\}} V_{\epsilon}(z) \leq\left(1+\zeta_{2}\right) W(r, 0) \text { for any } 0<r \leq \delta_{1} M_{\epsilon}^{\frac{2}{N-2 s}} \text { and } \epsilon \in\left(0, \epsilon_{2}\right) .
$$

Proof The proof is divided into three steps.

STEP 1 . We assert that for any parameter $0<\lambda<1$, there exists a large number $R=R(\lambda)>0$ such that

$$
\left(W-W_{\lambda^{2}, 0}\right)(z)>0 \text { for } \lambda<|z| \leq R .
$$

A direct computation with (2.13) shows that $w^{\lambda}(x)=w_{\lambda^{2}, 0}(x)$ for any $\lambda>0$ and $x \in \mathbb{R}^{N}$. By [30, Proposition 2.6] and the uniqueness of the $s$-harmonic extension, it follows that $W^{\lambda}=W_{\lambda^{2}, 0}$ in $\mathbb{R}_{+}^{N+1}$. Hence (3.4) and (7.1) imply that

$$
\begin{cases}\operatorname{div}\left(t^{1-2 s} \nabla\left(W-W_{\lambda^{2}, 0}\right)\right)=0 & \text { in } \mathbb{R}_{+}^{N+1}, \\ \left(W-W_{\lambda^{2}, 0}\right)(z)=\left(W-W^{\lambda}\right)(z)=0 & \text { on }|z|=\lambda \text { and } t>0, \\ \left(W-W_{\lambda^{2}, 0}\right)(z)>0 & \text { on }|z|=R \text { and } t>0, \\ \left(W-W_{\lambda^{2}, 0}\right)(x, 0)=\left(w-w_{\lambda^{2}, 0}\right)(x)>0 & \text { on } \lambda<|x| \leq R\end{cases}
$$

for some $R>0$ large. Now, the (classical) strong maximum principle justifies our claim (3.7).

We also notice that

$$
W(x, t) \leq w(x) \leq w(0)=\alpha_{N, s} \text { for }(x, t) \in \mathbb{R}_{+}^{N+1}
$$

where $\alpha_{N, s}>0$ is given in Notations.

STEP 2. From the definition (3.4), we have

$$
V_{\epsilon}^{\lambda}(z)=\left(\frac{\lambda}{|z|}\right)^{N-2 s} V_{\epsilon}\left(\frac{\lambda^{2} z}{|z|^{2}}\right) .
$$

By (3.3) and (3.8), there are values $\eta_{1}>0$ small and $R_{0}>0$ large such that 


$$
V_{\epsilon}^{\lambda}(z) \leq\left(1+\frac{\zeta_{2}}{4}\right) \alpha_{N, s}|z|^{-(N-2 s)} \text { for any } 0<\lambda \leq 1+\eta_{1} \text { and }|x| \leq R_{0},
$$

provided $\epsilon>0$ small enough. Let us take $\lambda_{1}=1-\eta_{1}$ and $\lambda_{2}=1+\eta_{1}$. Thanks to estimates (3.3) and (3.7), it is possible to select numbers $\eta_{2}>0$ small and $R_{1}>R_{0}$ large such that

$$
\begin{array}{lr}
D_{\epsilon}^{\lambda_{1}}(z)=V_{\epsilon}(z)-V_{\epsilon}^{\lambda_{1}}(z)>0 & \text { for } \lambda_{1}<|z| \leq R_{1}, \\
V_{\epsilon}^{\lambda_{1}}(z) \leq\left(1-2 \eta_{2}\right) \alpha_{N, s}|z|^{-(N-2 s)} & \text { for }|z| \geq R_{1}
\end{array}
$$

and

$$
\int_{B^{N}\left(0, R_{1}\right)} V_{\epsilon}^{p-\epsilon}(x, 0) \mathrm{d} x \geq\left(1-\frac{\eta_{2}}{2}\right) \int_{\mathbb{R}^{N}} w^{p}(x) \mathrm{d} x
$$

for any sufficiently small $\epsilon>0$.

Furthermore, we also have

$$
V_{\epsilon}(z) \geq\left(1-\eta_{2}\right) \alpha_{N, s}|z|^{-(N-2 s)} \quad \text { for } R_{1} \leq|z| \leq \delta_{1} M_{\epsilon}^{\frac{2}{N-2 s}}
$$

if $\delta_{1}>0$ is small enough. To verify it, let us choose a function $\hat{v}_{\epsilon}$ which solves

$$
(-\Delta)^{s} \hat{v}_{\epsilon}=V_{\epsilon}^{p-\epsilon}(\cdot, 0) \text { in } B^{N}\left(0, r_{0} M_{\epsilon}^{\frac{2}{N-2 s}}\right) \text { and } \hat{v}_{\epsilon}=0 \text { on } \partial B^{N}\left(0, r_{0} M_{\epsilon}^{\frac{2}{N-2 s}}\right) \text {, }
$$

and denote by $\widehat{V}_{\epsilon}$ its $s$-harmonic extension to the cylinder $B^{N}\left(0, r_{0} M_{\epsilon}^{\frac{2}{N-2 s}}\right) \times(0, \infty)$. Then, the comparison principle [25, Lemma 2.1] tells us that $V_{\epsilon} \geq \widehat{V}_{\epsilon}$. Since $H_{\mathcal{C}}^{1}(z, y)$ is bounded in $\left\{(z, y) \in \mathbb{R}_{+}^{N+1} \times \mathbb{R}^{N}:|z|,|y| \leq 1 / 2\right\}$, we obtain

$$
H_{\mathcal{C}}^{1}((x, t), y) \leq \frac{\eta_{2}}{4} \cdot \frac{\gamma_{N, s}}{|(x-y, t)|^{N-2 s}} \text { for }|(x, t)|,|y| \leq \frac{\delta_{1}}{r_{0}}
$$

by making $\delta_{1} \in\left(0, r_{0}\right)$ smaller if necessary. Moreover, because

$$
|(x-y, t)| \leq\left(1-\frac{1}{l}\right)|(x, t)| \text { for }|(x, t)| \geq l R_{1} \text { and }|y| \leq R_{1}
$$

given any large $l>1$, we see from (3.5), (3.12) and (3.14) that

$$
\begin{aligned}
\widehat{V}_{\epsilon}(x, t) & =\int_{B^{N}\left(0, r_{0} M_{\epsilon}^{\frac{2}{N-2 s}}\right)} V_{\epsilon}^{p-\epsilon}(y, 0) G_{\mathcal{C}}^{r_{0} M_{\epsilon}^{\frac{2}{N-2 s}}}((x, t), y) d y \\
& \geq\left(1-\frac{\eta_{2}}{4}\right) \int_{B^{N}\left(0, \delta_{1} M_{\epsilon}^{\frac{2}{N-2 s}}\right)} V_{\epsilon}^{p-\epsilon}(y, 0) \frac{\gamma_{N, s}}{|(x-y, t)|^{N-2 s}} d y \\
& \geq\left(1-\frac{\eta_{2}}{2}\right)\left(\int_{B^{N}\left(0, R_{1}\right)} V_{\epsilon}^{p-\epsilon}(y, 0) d y\right) \frac{\gamma_{N, s}}{|(x, t)|^{N-2 s}} \\
& \geq\left(1-\eta_{2}\right)\left(\int_{\mathbb{R}^{N}} w^{p}(y) d y\right) \frac{\gamma_{N, s}}{|(x, t)|^{N-2 s}} \\
& =\left(1-\eta_{2}\right) \frac{\alpha_{N, s}}{|(x, t)|^{N-2 s}} \quad \text { forl } R_{1} \leq|(x, t)| \leq \delta_{1} M_{\epsilon}^{\frac{2}{N-2 s}}
\end{aligned}
$$

by choosing $l$ large enough. If $R_{1} \leq|z| \leq l R_{1}$, we have $V_{\epsilon}(z) \geq\left(1-\eta_{2}\right) \alpha_{N, s}|z|^{-(N-2 s)}$ for $\epsilon>0$ small, for $V_{\epsilon}$ converges to $W$ uniformly over a compact set. This shows the validity of (3.13). 
STEP 3. Suppose that (3.6) does not hold with $\delta_{1}>0$ chosen in the previous step. Then

$$
\min _{\left\{z \in \mathbb{R}_{+}^{N+1}:|z|=r_{k}\right\}} V_{\epsilon_{k}}(z)>\left(1+\zeta_{2}\right) W\left(r_{k}, 0\right)
$$

for some sequences $\left\{\epsilon_{k}\right\}_{k \in \mathbb{N}}$ and $\left\{r_{k}\right\}_{k \in \mathbb{N}}$ of positive numbers such that $\epsilon_{k} \rightarrow 0$ and $r_{k} \in\left(0, \delta_{1} M_{\epsilon_{k}}^{\frac{2}{N-2 s}}\right)$. Because of (3.3), it should hold that $r_{k} \rightarrow \infty$. Thus Lemma 7.1 implies

$$
\min _{\left\{z \in \mathbb{R}_{+}^{N+1}:|z|=r_{k}\right\}} V_{k}(z) \geq\left(1+\frac{\zeta_{2}}{2}\right) \alpha_{N, s} r_{k}^{-(N-2 s)}
$$

where $V_{k}:=V_{\epsilon_{k}}$.

Now, we employ the method of moving spheres to the function $D_{k}^{\lambda}$ (see Remark 3.2 (2) for its definition). For any $k \in \mathbb{N}$ and $\mu \in\left[\lambda_{1}, \lambda_{2}\right]$, let

$$
\Sigma_{k}^{\mu}=\left\{x \in \overline{\mathbb{R}_{+}^{N+1}}: \mu<|z|<r_{k}\right\}
$$

and define a number $\bar{\lambda}_{k}$ by

$$
\bar{\lambda}_{k}=\sup \left\{\lambda \in\left[\lambda_{1}, \lambda_{2}\right]: D_{k}^{\mu}(z) \geq 0 \text { in } \Sigma_{k}^{\mu} \text { for all } \lambda_{1} \leq \mu \leq \lambda\right\} .
$$

By (3.11) and (3.13), we see that $\bar{\lambda}_{k} \geq \lambda_{1}$. We shall show that $\bar{\lambda}_{k}=\lambda_{2}$ for sufficiently large $k \in \mathbb{N}$.

To the contrary, assume that $\bar{\lambda}_{k}<\lambda_{2}$ for some large fixed index $k \in \mathbb{N}$. By continuity it holds that $D_{k}^{\bar{\lambda}_{k}} \geq 0$ in $\Sigma_{k}^{\bar{\lambda}_{k}}$. Moreover, from (3.16) and (3.10), we have $D_{k}^{\bar{\lambda}_{k}}>0$ on $\left\{z \in \mathbb{R}_{+}^{N+1}:|z|=r_{k}\right\}$, which implies that $D_{k}^{\bar{\lambda}_{k}} \neq 0$ in $\Sigma_{k}^{\bar{\lambda}_{k}}$. Thus, it holds that $D_{k}^{\bar{\lambda}_{k}}>0$ in $\Sigma_{k}^{\bar{\lambda}_{k}}$ thanks to the strong maximum principle. Pick $\delta>0$ small so that the maximum principle for domains with small volume [30, Lemma 2.8] can be applied. If we choose a compact set $K \subset \Sigma_{k}^{\bar{\lambda}_{k}}$ such that $\left|\Sigma_{k}^{\bar{\lambda}_{k}} \backslash K\right|<\delta$, then $\inf _{K} D_{k}^{\bar{\lambda}_{k}}>0$. By continuity again, for $\lambda \in\left(\bar{\lambda}_{k}, \lambda_{2}\right)$ sufficiently close to $\bar{\lambda}_{k}$, we have

$$
K \subset \Sigma_{k}^{\lambda}, \quad\left|\Sigma_{k}^{\lambda} \backslash K\right|<\delta \text { and } \inf _{K} D_{k}^{\lambda}>0 .
$$

Consequently, we see from [30, Lemma 2.8] that $D_{k}^{\lambda} \geq 0$, contradicting the maximality of $\bar{\lambda}_{k}$. Therefore, it should hold that $\bar{\lambda}=\lambda_{2}$.

Finally, taking a limit $k \rightarrow \infty$ to $D_{k}^{\lambda_{2}} \geq 0$ in $\Sigma_{k}^{\lambda_{2}}$, we get

$$
W(z) \geq W^{\lambda_{2}}(z) \text { in }|z| \geq \lambda_{2} \text {. }
$$

However, it is impossible since $\lambda_{2}>1$. Therefore, (3.6) should be true.

We now complete the proof of Proposition 3.1.

Lemma 3.4 Let $\left\{V_{\epsilon}\right\}_{\epsilon>0}$ be the family in the statement of Proposition 3.1 and $\delta_{1}>0$ the number selected in the proof of the previous lemma. Then, there exist a constant $C>0$ and small parameter $\delta_{0} \in\left(0, \delta_{1}\right)$ such that

$$
V_{\epsilon}(z) \leq C W(z) \text { for all } z \in B_{+}^{N+1}\left(0, \delta_{0} M_{\epsilon}^{\frac{2}{N-2 s}}\right)
$$

provided that $\epsilon>0$ is sufficiently small. 
Proof By virtue of lemmas 3.3 and 7.1, we have a point $z_{0}=\left(x_{0}, t_{0}\right) \in \mathbb{R}_{+}^{N+1}$ such that $\left|z_{0}\right|=\delta_{2} M_{\epsilon}^{\frac{2}{N-2 s}}$ and

$$
V_{\epsilon}\left(z_{0}\right) \leq\left(1+\zeta_{2}\right) W\left(\left|z_{0}\right|, 0\right) \leq\left(1+2 \zeta_{2}\right) \alpha_{N, s}\left|z_{0}\right|^{-(N-2 s)}
$$

for any small $\delta_{2} \in\left(0, \delta_{1}\right)$. Let $G_{\mathcal{C}}^{*}$ be Green's function of (2.7) in the semi-infinite cylinder $\mathcal{C}=B^{N}\left(0, \delta_{1} M_{\epsilon}^{\frac{2}{N-2 s}}\right) \times(0, \infty)($ refer to Remark $3.2(3))$. Then, we are able to choose a constant $\delta_{3} \in\left(0, \delta_{2}\right)$ so small that

$$
\begin{aligned}
V_{\epsilon}\left(z_{0}\right) & \geq \int_{B^{N}\left(0, \delta_{1} M_{\epsilon} \frac{2}{N-2 s}\right)} V_{\epsilon}^{p-\epsilon}(y, 0) G^{*}\left(z_{0}, y\right) d y \\
& \geq\left(1-\zeta_{2}\right) \gamma_{N, s} \int_{B^{N}\left(0, \delta_{2} M_{\epsilon}^{\frac{N}{N-2 s}}\right)} V_{\epsilon}^{p-\epsilon}(y, 0) \frac{1}{\left|\left(x_{0}-y, t_{0}\right)\right|^{N-2 s}} d y \\
& \geq\left(1-2 \zeta_{2}\right) \gamma_{n, s}\left|z_{0}\right|^{-(N-2 s)} \int_{B^{N}\left(0, \delta_{3} M_{\epsilon}^{\frac{2}{N-2 s}}\right)} V_{\epsilon}^{p-\epsilon}(y, 0) d y
\end{aligned}
$$

as in (3.15). Combining the above two estimates with (3.12), we obtain

$$
\int_{B^{N}\left(0, \delta_{3} M_{\epsilon}^{\frac{2}{N-2 s}}\right) \backslash B^{N}\left(0, R_{1}\right)} V_{\epsilon}^{p-\epsilon}(y, 0) d y \leq C \zeta_{2} .
$$

Since $V_{\epsilon}$ is uniformly bounded, we observe from (3.17) that

$$
\int_{B^{N}\left(0, \delta_{3} M_{\epsilon}^{N-2 s}\right) \backslash B^{N}\left(0, R_{1}\right)} V_{\epsilon}^{p+1}(y, 0) d y \leq C \zeta_{2} .
$$

Now, let us define $V_{r, \epsilon}(z)=r^{\frac{N-2 s}{2}} V_{\epsilon}(r z)$ on the half-annulus $\left\{z \in \mathbb{R}_{+}^{N+1}: 1 / 2 \leq|z| \leq 2\right\}$ for each $2 R_{1} \leq r \leq \delta_{3} M_{\epsilon}^{\frac{2}{N-2 s}} / 2$ and $\epsilon>0$ small. Then, one can apply the Moser iteration method with (3.18) (refer to [25]) to deduce that it is uniformly bounded in $\left\{z \in \mathbb{R}_{+}^{N+1}\right.$ : $3 / 4 \leq|z| \leq 3 / 2\}, r$ and $\epsilon$. As a result, the Harnack inequality [12, Lemma 4.9] yields

$$
\sup _{\left\{z \in \mathbb{R}_{+}^{N+1}: 3 / 4 \leq|z| \leq 3 / 2\right\}} V_{r, \epsilon}(z) \leq C \inf _{\left\{z \in \mathbb{R}_{+}^{N+1}: 3 / 4 \leq|z| \leq 3 / 2\right\}} V_{r, \epsilon}(z)
$$

where $C>0$ is a universal constant. This inequality with Lemma 3.3 and (3.3) concludes the proof of the lemma (giving $\delta_{0}=3 \delta_{3} / 4$ ).

The following assertion is an immediate consequence of Proposition 3.1.

Corollary 3.5 Fix any $x_{0} \in \mathbb{R}^{N}$ and small $r_{0}>0$. Let $\left\{U_{\epsilon}\right\}_{\epsilon>0}$ be a family of positive solutions to

$$
\begin{cases}\operatorname{div}\left(t^{1-2 s} \nabla U_{\epsilon}\right)=0 & \text { in } B^{N}\left(x_{0}, r_{0}\right) \times(0, \infty), \\ \partial_{\nu}^{s} U_{\epsilon}=U_{\epsilon}^{p-\epsilon} & \text { on } B^{N}\left(x_{0}, r_{0}\right), \\ \left\|U_{\epsilon}\right\|_{L^{\infty}\left(B_{+}^{N+1}\left(\left(x_{0}, 0\right), r_{0}\right)\right)} \leq c M_{\epsilon}^{\frac{N-2 s}{2}} & \end{cases}
$$

for a certain constant $c>0$ independent of $\epsilon$ and a family of positive values $\left\{M_{\epsilon}\right\}_{\epsilon>0}$ such that $\lim _{\epsilon \rightarrow \infty} M_{\epsilon}=\infty$ and $\lim _{\epsilon \rightarrow \infty} M_{\epsilon}^{\epsilon}=1$. Suppose that the rescaled function $M_{\epsilon}^{-\frac{N-2 s}{2}} U_{\epsilon}\left(M_{\epsilon}^{-1} \cdot+\left(x_{0}, 0\right)\right)$ converges weakly to the function $W$ in $D^{1,2}\left(\mathbb{R}_{+}^{N+1} ; t^{1-2 s}\right)$. Then, we have

$$
U_{\epsilon}(z) \leq C M_{\epsilon}^{\frac{N-2 s}{2}} W\left(M_{\epsilon}\left(z-\left(x_{0}, 0\right)\right)\right) \text { for all } z \in B_{+}^{N+1}\left(\left(x_{0}, 0\right), \delta_{0}\right)
$$


for some $\delta_{0} \in\left(0, r_{0}\right)$ and $C>0$ independent of $\epsilon$.

\section{Application of the pointwise upper estimate}

In this section, we gather refined information on finite energy solutions $U_{\epsilon}$ to Eq. (2.4). More precisely, we first show that $V_{0}$ vanishes identically if $m \neq 0$ in (2.19). Then, we prove that any two different blow-up points do not collide and blow-up rates of each bubble are compatible to the others. Finally, we get sharp pointwise upper bounds of $U_{\epsilon}$ over the whole cylinder $\mathcal{C}$ and deduce that a suitable $L^{\infty}$-normalization of $U_{\epsilon}$ converges to a certain function as $\epsilon \searrow 0$, which can be described as a combination of Green's function.

Recall from (2.19), (2.22) and (2.23) that

$$
U_{n}=V_{0}+\sum_{i=1}^{m} P W_{\lambda_{n}^{i}, x_{n}^{i}}+R_{n} \text { in } \mathcal{C}^{\prime}
$$

and $x_{0}^{i}=\lim _{n \rightarrow \infty} x_{n}^{i} \in \Omega$ for each $i=1, \ldots, m$. We also remind with (2.21) that the concentration rate $\lambda_{n}^{i}$ on each blow-up part tends to 0 as $n \rightarrow \infty$. The next lemma ensures that this convergence is not too fast.

Lemma 4.1 Let $\left\{U_{n}\right\}_{n \in \mathbb{N}}$ be a sequence of solutions to (2.4) with $\epsilon=\epsilon_{n} \searrow 0$, which admits a decomposition of the form (4.1). Then, we have $\lim _{n \rightarrow \infty}\left(\lambda_{n}^{i}\right)^{\epsilon_{n}}=1$ for each $1 \leq i \leq m$.

Proof Fix any $i \in\{1, \ldots, m\}$. Multiplying (2.4) by $P W_{\lambda_{n}^{i}, x_{n}^{i}}$, integrating by parts and using (2.15), we get the equality

$$
\int_{\Omega} u_{n}^{p-\epsilon_{n}} P w_{\lambda_{n}^{i}, x_{n}^{i}} \mathrm{~d} x=\kappa_{s} \int_{\mathcal{C}} t^{1-2 s} \nabla U_{n} \cdot \nabla P W_{\lambda_{n}^{i}, x_{n}^{i}} \mathrm{~d} x \mathrm{~d} t=\int_{\Omega} u_{n} w_{\lambda_{n}^{i}, x_{n}^{i}}^{p} \mathrm{~d} x .
$$

Let us estimate the leftmost and rightmost sides of (4.2). By making use of (4.1), (2.21), the mean value theorem, and the fact that $v_{0}$ is bounded on $\Omega \times\{0\}$ and $\lim _{n \rightarrow \infty}\left\|R_{n}\right\|_{H_{0}^{1,2}\left(\mathcal{C} ; t^{1-2 s}\right)}=0$, we obtain

$$
\begin{aligned}
& \int_{\Omega}\left|\left(u_{n}^{p-\epsilon_{n}}-\left(P w_{\lambda_{n}^{i}, x_{n}^{i}}\right)^{p-\epsilon_{n}}\right) P w_{\lambda_{n}^{i}, x_{n}^{i}}\right| \mathrm{d} x \\
& \leq C \int_{\Omega}\left|\sum_{j \neq i} P w_{\lambda_{n}^{j}, x_{n}^{j}}+v_{0}+r_{n}\right|\left(\sum_{j=1}^{m}\left(P w_{\lambda_{n}^{j}, x_{n}^{j}}\right)^{p-1-\epsilon_{n}}+\left|v_{0}\right|^{p-1-\epsilon_{n}}+\left|r_{n}\right|^{p-1-\epsilon_{n}}\right) \\
& \quad P w_{\lambda_{n}^{i}, x_{n}^{i}} \mathrm{~d} x=o(1) .
\end{aligned}
$$

Hence, it holds

$$
\int_{\Omega} u_{n}^{p-\epsilon_{n}} P w_{\lambda_{n}^{i}, x_{n}^{i}} \mathrm{~d} x=\int_{\Omega}\left(P w_{\lambda_{n}^{i}, x_{n}^{i}}\right)^{p+1-\epsilon_{n}} \mathrm{~d} x+o(1) .
$$

Moreover, it is easy to check that

$$
\begin{aligned}
\int_{\Omega}\left(P w_{\lambda_{n}^{i}, x_{n}^{i}}\right)^{p+1-\epsilon_{n}} \mathrm{~d} x & =\left(\lambda_{n}^{i}\right)^{-\left(\frac{N-2 s}{2}\right) \epsilon_{n}} \int_{\lambda_{n}^{i}\left(\Omega-x_{n}^{i}\right)}\left(P w_{1,0}\right)^{p+1-\epsilon_{n}} \mathrm{~d} x \\
& =\left(\lambda_{n}^{i}\right)^{-\left(\frac{N-2 s}{2}\right) \epsilon_{n}}\left(\int_{\mathbb{R}^{N}} w^{p+1} \mathrm{~d} x+o(1)\right) .
\end{aligned}
$$


Similarly, one may show that

$$
\int_{\Omega} u_{n} w_{\lambda_{n}^{i}, x_{n}^{i}}^{p} \mathrm{~d} x=\int_{\mathbb{R}^{N}} w^{p+1} \mathrm{~d} x+o(1) .
$$

Substituting (4.3), (4.4) and (4.5) into (4.2), we conclude that $\lim _{n \rightarrow \infty}\left(\lambda_{n}^{i}\right)^{\epsilon_{n}}=1$. The lemma is proved.

In the following, we give the proof of several claims stated in the beginning of this section, applying the previous lemma.

Lemma 4.2 Let $\left\{U_{n}\right\}_{n \in \mathbb{N}}$ be a sequence of solutions of (2.4) with $\epsilon=\epsilon_{n}$ which admits an asymptotic behavior (4.1). Suppose that there exists at least one bubble in (4.1), i.e., $m \neq 0$. Then, $V_{0} \equiv 0$.

Proof Firstly, we aim to show that

$$
U_{n}(z) \leq C\left(\lambda_{n}^{1}\right)^{-\frac{N-2 s}{2}} \text { uniformly for any } z \in \mathcal{C} \text { and } n \in \mathbb{N} .
$$

To do so, we consider the function $\widetilde{U}_{n}(z):=\left(\lambda_{n}^{1}\right)^{\frac{N-2 s}{2}} U_{n}\left(\lambda_{n}^{1} z\right)$ defined in $\mathcal{C}_{n}:=\left(\lambda_{n}^{1}\right)^{-1} \mathcal{C}$. One can easily observe that it satisfies

$$
\begin{cases}\operatorname{div}\left(t^{1-2 s} \nabla \widetilde{U}_{n}\right)=0 & \text { in } \mathcal{C}_{n}, \\ \widetilde{U}_{n}=0 & \text { on } \partial_{L} \mathcal{C}_{n}, \\ \partial_{\nu}^{s} \widetilde{U}_{n}=\left(\lambda_{n}^{1}\right)^{\frac{(N-2 s) \epsilon}{2}} \widetilde{U}_{n}^{p-\epsilon} & \text { on } \Omega_{n} \times\{0\}\end{cases}
$$

where $\Omega_{n}:=\left(\lambda_{n}^{1}\right)^{-1} \Omega$. Also it is plain to check

$$
\sup _{n \in \mathbb{N}} \int_{\mathcal{C}_{n}} t^{1-2 s}\left|\nabla \widetilde{U}_{n}(x, t)\right|^{2} \mathrm{~d} x \mathrm{~d} t<C \text { and } \sup _{n \in \mathbb{N}} \int_{\Omega_{n}}\left|\widetilde{U}_{n}(x, 0)\right|^{\frac{2 N}{N-2 s}} \mathrm{~d} x<C .
$$

Owing to Hölder's inequality, it holds that

$$
\sup _{n \in \mathbb{N}} \int_{B^{N}\left(y, r_{0}\right) \cap \Omega_{n}}\left|\widetilde{U}_{n}(x, 0)\right|^{2} \mathrm{~d} x<C
$$

for any $y \in \Omega_{n}$ and a small value $r_{0}>0$ to be fixed soon. Combining this with the first estimate of (4.7) yields

$$
\sup _{n \in \mathbb{N}} \int_{B_{+}^{N+1}\left((y, 0), r_{0}\right) \cap \mathcal{C}_{n}} t^{1-2 s}\left|\widetilde{U}_{n}(x, t)\right|^{2} \mathrm{~d} x \mathrm{~d} t<C
$$

(see the proof of [24, Lemma 3.1]). Let $\delta>0$ be the number in Lemma 8.1. Then, from (2.22), (4.1) and the fact that

$$
\lim _{n \rightarrow \infty} \int_{\Omega_{n}}\left|\left(\lambda_{n}^{1}\right)^{\frac{N-2 s}{2}} R_{n}\left(\lambda_{n}^{1} x, 0\right)\right|^{\frac{2 N}{N-2 s}} \mathrm{~d} x=0,
$$

it is possible to choose $r_{0}>0$ small enough so that

$$
\sup _{n \in \mathbb{N}} \int_{B^{N}\left(y, r_{0}\right) \cap \Omega_{n}}\left|\widetilde{U}_{n}(x, 0)\right|^{\frac{2 N}{N-2 s}} \mathrm{~d} x<\delta .
$$

Therefore, by invoking Lemma 8.1 with $a=\left(\lambda_{n}^{1}\right)^{\frac{(N-2 s) \epsilon}{2}} \widetilde{U}_{n}^{p-1-\epsilon}$ and $f=0$, we may conclude that

$$
\sup _{n \in \mathbb{N}}\left\|\widetilde{U}_{n}\right\|_{L^{\infty}\left(B^{N}\left(y, r_{0} / 2\right) \cap \Omega_{n}\right)} \leq C \sup _{n \in \mathbb{N}} \int_{B_{+}^{N+1}\left((y, 0), r_{0}\right) \cap \mathcal{C}_{n}} t^{1-2 s}\left|\widetilde{U}_{n}(x, t)\right|^{2} \mathrm{~d} x \mathrm{~d} t \leq C
$$


where the last inequality is due to (4.8). Since $y \in \Omega_{n}$ is chosen arbitrarily and $\widetilde{U}_{n}$ attains its maximum on $\Omega_{n} \times\{0\}$, it follows

$$
\sup _{n \in \mathbb{N}} \sup _{(x, t) \in \mathcal{C}_{n}} \widetilde{U}_{n}(x, t)=\sup _{n \in \mathbb{N}} \sup _{x \in \Omega_{n}} \widetilde{U}_{n}(x, 0) \leq C .
$$

This proves (4.6).

Now, by virtue of (4.6), Corollary 3.5 and Lemma 4.1, we obtain

$$
U_{n}(z) \leq C\left(\lambda_{n}^{1}\right)^{-\frac{N-2 s}{2}} W\left(\frac{z-\left(x_{n}^{1}, 0\right)}{\lambda_{n}^{1}}\right) \text { for all } z \in B_{+}^{N+1}\left(\left(x_{n}^{1}, 0\right), \delta_{0}\right),
$$

which implies

$$
\lim _{n \rightarrow \infty} U_{n}(z)=0 \text { for any } z \in B_{+}^{N+1}\left(\left(x_{0}^{1}, 0\right), \delta_{0} / 2\right) \backslash\left\{\left(x_{0}^{1}, 0\right)\right\} .
$$

Since $R_{n}(\cdot, 0) \rightarrow 0$ in $L^{\frac{2 N}{N-2 s}}(\Omega)$, there exists a point $x^{\prime} \in B^{N}\left(x_{0}^{1}, \delta_{0} / 2\right) \backslash\left\{x_{0}^{1}, \ldots, x_{0}^{m}\right\}$ such that $\lim _{n \rightarrow \infty} R_{n}\left(x^{\prime}, 0\right)=0$. Furthermore, we know from (4.1) that $U_{n}(x, 0) \geq V_{0}(x, 0)+$ $R_{n}(x, 0)$ for all $x \in \Omega$, so it should hold that $V_{0}\left(x^{\prime}, 0\right)=0$.

On the other hand, each $U_{n}$ and its weak limit $V_{0}$ are nonnegative in $\mathcal{C}$. Therefore, one concludes from the strong maximum principle that $V_{0} \equiv 0$.

In Lemmas 4.3-4.6, we are mainly interested in the case $m \neq 0$. In this situation, solutions $U_{n}$ to (2.4) with the asymptotic behavior (4.1) can be rewritten in the form

$$
U_{n}=\sum_{i=1}^{m} P W_{\lambda_{n}^{i}, x_{n}^{i}}+R_{n} \text { in } \mathcal{C}^{\prime}
$$

where $\lim _{n \rightarrow \infty}\left\|R_{n}\right\|_{H_{0}^{1,2}\left(\mathcal{C} ; t^{1-2 s}\right)}=0$.

Lemma 4.3 Assume that a sequence $\left\{U_{n}\right\}_{n \in \mathbb{N}}$ of solutions to (2.4) with $\epsilon=\epsilon_{n}$ has the asymptotic behavior given by Lemma 2.2 with $m \geq 1$. Then, there exists a constant $d_{0}>0$ such that

$$
\left|x_{0}^{i}-x_{0}^{j}\right| \geq d_{0} \text { for any } 1 \leq i<j \leq m .
$$

Proof Assume that two different blow-up points converge to the same point $x^{\prime} \in \Omega$. By (2.21) and (2.22), one of the following holds:

$$
\text { (1) } \lim _{n \rightarrow \infty} \frac{\lambda_{n}^{i}}{\lambda_{n}^{j}}=0 \text { or (2) } \lim _{n \rightarrow \infty} \frac{\left|x_{n}^{i}-x_{n}^{j}\right|^{2}}{\lambda_{n}^{i} \lambda_{n}^{j}}=\infty \text {. }
$$

Suppose that (1) holds. Then by (2.22), it should be true that

$$
\lim _{n \rightarrow \infty} \frac{\lambda_{n}^{1}}{\lambda_{n}^{m}}=0 .
$$

We shall prove that it cannot happen. By Corollary 3.5, we have an upper bound (4.9). Furthermore, we can find a lower bound

$$
U_{n}(z) \geq C\left(\lambda_{n}^{m}\right)^{\frac{N-2 s}{2}} \text { for all } z \in B_{+}^{N+1}\left(\left(x^{\prime}, 0\right), \delta_{0}\right)
$$

where $\delta_{0}>0$ is a number in (4.9) (taken smaller if required). Indeed, by (2.18), (2.19), (2.21) and Lemma 4.2, we have

$$
\left(\lambda_{n}^{m}\right)^{\frac{N-2 s}{2}} u_{n}\left(\lambda_{n}^{m} y+x_{n}^{m}\right) \rightarrow w(y) \text { for a.e. } y \in \mathbb{R}^{N} .
$$


Thus Green's representation formula, Fatou's lemma and Lemma 4.1 show

$$
\begin{aligned}
U_{n}(z) & \geq \int_{B^{N}\left(x_{n}^{m}, \delta_{0}\right)} G_{\mathcal{C}}(z, x) u_{n}^{p-\epsilon_{n}}(x) \mathrm{d} x \geq C \int_{B^{N}\left(x_{n}^{m}, \delta_{0}\right)} u_{n}^{p-\epsilon_{n}}(x) \mathrm{d} x \\
& =C\left(\lambda_{n}^{m}\right)^{\frac{N-2 s}{2}\left(1+\epsilon_{n}\right)} \int_{B^{N}\left(0, \delta_{0} / \lambda_{n}^{m}\right)}\left[\left(\lambda_{n}^{m}\right)^{\frac{N-2 s}{2}} u_{n}\left(\lambda_{n}^{m} y+x_{n}^{m}\right)\right]^{p-\epsilon_{n}} d y \\
& \geq C\left(\int_{\mathbb{R}^{N}} w^{p}(y) d y+o(1)\right)\left(\lambda_{n}^{m}\right)^{\frac{N-2 s}{2}},
\end{aligned}
$$

which confirms (4.13). Now fixing any point $z^{*} \in \mathbb{R}_{+}^{N+1}$ such that $\left|z^{*}-\left(x^{\prime}, 0\right)\right|=\delta_{0} / 2$ and putting it into (4.9) and (4.13), we discover that $\left(\lambda_{n}^{m}\right)^{\frac{N-2 s}{2}} \leq C\left(\lambda_{n}^{1}\right)^{\frac{N-2 s}{2}}$ for some $C>0$, contradicting (4.12). Therefore (1) is false, and we may assume that

$$
\lim _{n \rightarrow \infty} \frac{\lambda_{n}^{i}}{\lambda_{n}^{j}}=c_{0} \quad \text { for some } c_{0} \in(0,1] \text {. }
$$

Assume that (2) is true. Owing to (4.15), inequality (4.6) can be written as

$$
U_{n}(z) \leq C\left(\lambda_{n}^{j}\right)^{-\frac{N-2 s}{2}} \leq C\left(\lambda_{n}^{i}\right)^{-\frac{N-2 s}{2}} \text { for } z \in \mathcal{C} \text { and } n \in \mathbb{N} .
$$

Hence, we infer from elliptic regularity and Corollary 3.5 that

$$
\left(\lambda_{n}^{j}\right)^{\frac{N-2 s}{2}} u_{n}\left(\lambda_{n}^{j} \cdot+x_{n}^{j}\right) \rightarrow w \text { in } C^{\alpha}\left(\mathbb{R}^{N}\right) \text { for some } \alpha \in(0,1)
$$

and

$$
U_{n}(z) \leq C\left(\lambda_{n}^{i}\right)^{-\frac{N-2 s}{2}} W\left(\frac{z-\left(x_{n}^{j}, 0\right)}{\lambda_{n}^{i}}+\frac{\left(x_{n}^{j}-x_{n}^{i}, 0\right)}{\lambda_{n}^{i}}\right)
$$

for all $z \in B_{+}^{N+1}\left(\left(x^{\prime}, 0\right), \delta_{0} / 2\right)$ and large $n \in \mathbb{N}$. Since $\lim _{n \rightarrow \infty}\left|x_{n}^{j}-x_{n}^{i}\right| / \lambda_{n}^{i}=\infty$ holds because of (2.22), if we take $z=\left(x_{n}^{j}, 0\right)$ in inequality (4.17) and use (4.16), then we get

$$
C\left(\lambda_{n}^{j}\right)^{-\frac{N-2 s}{2}} \leq u_{n}\left(x_{n}^{j}\right) \leq C\left(\lambda_{n}^{i}\right)^{-\frac{N-2 s}{2}} w\left(\frac{x_{n}^{j}-x_{n}^{i}}{\lambda_{n}^{i}}\right)=o(1) \cdot\left(\lambda_{n}^{i}\right)^{-\frac{N-2 s}{2}}
$$

provided $n \in \mathbb{N}$ large. However, this is absurd as (4.15) holds, and so (2) does not hold either.

Summing up, every possible case is excluded if two blow-up points tend to the same point. Accordingly, (4.11) has the validity.

In the following lemma, we study the behavior of solutions $u_{n}$ to (1.1) outside the blow-up points $\left\{x_{0}^{1}, \ldots, x_{0}^{m}\right\}$. We set

$$
A_{r}=\Omega \backslash \bigcup_{i=1}^{m} B^{N}\left(x_{0}^{i}, r\right) \text { for any } r>0 .
$$

Lemma 4.4 Suppose that $\left\{U_{n}\right\}_{n \in \mathbb{N}}$ is a family of solutions for (2.4) with $\epsilon=\epsilon_{n}$ satisfying the asymptotic behavior (4.10). Then, for any small $r>0$, we have $u_{n}(x)=O\left(\left(\lambda_{n}^{m}\right)^{\frac{N-2 s}{2}}\right)$ uniformly for $x \in A_{r}$. 
Proof Let $a_{n}=u_{n}^{p-1-\epsilon_{n}}$ so that $\partial_{v}^{s} U_{n}=a_{n} u_{n}$ in $\Omega \times\{0\}$. Then, we see from (1.5) that $\left\|a_{n}\right\|_{L^{\frac{N}{2 s}}\left(A_{r / 4}\right)} \leq C\left(\sum_{i=1}^{m}\left\|w_{\lambda_{n}^{i}, x_{n}^{i}}\right\|_{L^{p+1-\left(\frac{N}{2 s}\right) \epsilon_{n}}\left(\mathbb{R}^{N} \backslash B^{N}\left(x_{0}^{i}, r / 4\right)\right)}^{p-1-\epsilon_{n}}+\left\|R_{n}\right\|_{H_{0}^{1,2}\left(\mathcal{C} ; t^{1-2 s}\right)}\right)=o(1)$.

Therefore, we can proceed the Moser iteration argument to get $\left\|a_{n}\right\|_{L^{q}\left(A_{r / 2}\right)}=o(1)$ for some $q>\frac{N}{2 s}$, and it further leads to $\left\|u_{n}\right\|_{L^{\infty}\left(A_{r}\right)}=o(1)$ (see Sect. 3 in [25]).

Assume that $r \in\left(0, \min \left\{\delta_{0}, d_{0} / 2\right\}\right)$ where $\delta_{0}>0$ and $d_{0}$ are the numbers picked up in Corollary 3.5 and Lemma 4.3, respectively. Then, the argument used to derive (4.6) with Lemma 4.3 deduces

$$
U_{n}(x, t) \leq C\left(\lambda_{n}^{i}\right)^{-\frac{N-2 s}{2}} \text { for }\left|x-x_{0}^{i}\right| \leq r \text { and } t \geq 0
$$

so that Corollary 3.5 implies

$$
u_{n}(x) \leq C\left(\lambda_{n}^{i}\right)^{-\frac{N-2 s}{2}} w\left(\frac{x-x_{n}^{i}}{\lambda_{n}^{i}}\right) \leq C\left(\lambda_{n}^{i}\right)^{\frac{N-2 s}{2}} \text { for } \frac{r}{2} \leq\left|x-x_{0}^{i}\right| \leq r
$$

where $i=1, \ldots, m$. By Green's representation formula, one may write

$$
u_{n}(x)=\int_{A_{r / 2}} G(x, y) u_{n}^{p-\epsilon_{n}}(y) d y+\sum_{i=1}^{m} \int_{B^{N}\left(x_{0}^{i}, r / 2\right)} G(x, y) u_{n}^{p-\epsilon_{n}}(y) d y .
$$

If we set $b_{n}=\left\|u_{n}\right\|_{L^{\infty}\left(A_{r}\right)}$, then we observe with assumption (2.22) that

$$
\begin{aligned}
& \int_{A_{r / 2}} G(x, y) u_{n}^{p-\epsilon_{n}}(y) d y \\
& \quad \leq C \int_{A_{r / 2}} G(x, y)\left(b_{n}^{p-\epsilon_{n}}+\max \left\{\lambda_{n}^{1}, \ldots, \lambda_{n}^{m}\right\}^{\frac{N-2 s}{2}\left(p-\epsilon_{n}\right)}\right) d y \\
& \quad \leq C\left(b_{n}^{p-\epsilon_{n}}+\left(\lambda_{n}^{m}\right)^{\frac{N-2 s}{2}\left(p-\epsilon_{n}\right)}\right)
\end{aligned}
$$

for any $x \in A_{r}$. Besides, Corollary 3.5 and Lemma 4.1 give us that

$$
\begin{aligned}
& \int_{B^{N}\left(x_{0}^{i}, r / 2\right)} G(x, y) u_{n}^{p-\epsilon_{n}}(y) d y \\
& \quad \leq C \int_{B^{N}\left(x_{0}^{i}, r / 2\right)} u_{n}^{p-\epsilon_{n}}(y) d y \\
& \quad \leq C \int_{B^{N}\left(x_{0}^{i}, r / 2\right)} w_{\lambda_{n}^{i}, x_{n}^{i}}^{p-\epsilon_{n}}(y) d y \leq C\left(\lambda_{n}^{i}\right)^{\frac{N-2 s}{2}}
\end{aligned}
$$

for all $x \in A_{r}$ and each $i=1, \ldots, m$. Hence, by combining (4.19) and (4.20), we get

$$
b_{n} \leq C\left(b_{n}^{p-\epsilon_{n}}+\left(\lambda_{n}^{m}\right)^{\frac{N-2 s}{2}}\right) \text {. }
$$

Since we have $p-\epsilon_{n}>1$ and $b_{n}=o(1)$, the above inequality implies that $b_{n} \leq C\left(\lambda_{n}^{m}\right)^{\frac{N-2 s}{2}}$. The lemma is proved.

We prove the compatibility of the blow-up rates $\left\{\lambda_{n}^{1}, \ldots, \lambda_{n}^{m}\right\}$. 
Lemma 4.5 There exists a constant $C_{0}>0$ independent of $n \in \mathbb{N}$ such that

$$
\frac{\lambda_{n}^{i}}{\lambda_{n}^{j}} \leq C_{0} \text { for any } 1 \leq i, j \leq m
$$

Proof As in (4.14), it can be verified that $u_{n}(x) \geq C\left(\lambda_{n}^{i}\right)^{\frac{N-2 s}{2}}$ in $\bigcup_{k=1}^{m} B^{N}\left(x_{0}^{k}, r\right)$ for each $i=1, \ldots, m$. As a matter of fact, it is possible to substitute $x_{n}^{m}$ and $\lambda_{n}^{m}$ in (4.14) with $x_{n}^{i}$ and $\lambda_{n}^{i}$, respectively.

On the other hand, we know from Lemma 4.4 that $u_{n}(x) \leq C\left(\lambda_{n}^{j}\right)^{\frac{N-2 s}{2}}$ for $x \in$ $B^{N}\left(x_{0}^{j}, r\right) \backslash B^{N}\left(x_{0}^{j}, r / 2\right)$. Thus, we have $\left(\lambda_{n}^{i}\right)^{\frac{N-2 s}{2}} \leq C\left(\lambda_{n}^{j}\right)^{\frac{N-2 s}{2}}$ for any $1 \leq i, j \leq m$. The proof is done.

As in the statement of Theorem 1.1, we set $b_{i}=\lim _{n \rightarrow \infty}\left(\frac{\lambda_{n}^{i}}{\lambda_{n}^{1}}\right)^{\frac{N-2 s}{2}} \in(0, \infty)$ for any $i=1, \ldots, m$.

Lemma 4.6 Suppose that $\left\{U_{n}\right\}_{n \in \mathbb{N}}$ is a sequence of solutions to Eq. (2.4) with $\epsilon=\epsilon_{n}$ which admit the asymptotic behavior (4.10). Then, it holds

$$
\lim _{n \rightarrow \infty}\left(\lambda_{n}^{1}\right)^{-\frac{N-2 s}{2}} U_{n}(x, t)=c_{1} \sum_{i=1}^{m} b_{i} G_{\mathcal{C}}\left((x, t), x_{0}^{i}\right)
$$

in $C^{0}\left(\mathcal{C}^{\prime} \backslash\left\{\left(x_{0}^{1}, 0\right), \ldots,\left(x_{0}^{m}, 0\right)\right\}\right)$. Furthermore, we have

$$
\lim _{n \rightarrow \infty}\left(\lambda_{n}^{1}\right)^{-\frac{N-2 s}{2}} \nabla_{x}^{k} U_{n}(x, t)=c_{1} \sum_{i=1}^{m} b_{i} \nabla_{x}^{k} G_{\mathcal{C}}\left((x, t), x_{0}^{i}\right)
$$

for $1 \leq k \leq 2$ and

$$
\lim _{n \rightarrow \infty}\left(\lambda_{n}^{1}\right)^{-\frac{N-2 s}{2}} t^{l-2 s} \partial_{t}^{l} \nabla_{x}^{k} U_{n}(x, t)=c_{1} \sum_{i=1}^{m} b_{i} t^{l-2 s} \partial_{t}^{l} \nabla_{x}^{k} G_{\mathcal{C}}\left((x, t), x_{0}^{i}\right)
$$

for any pair $(k, l)$ such that $0 \leq k \leq 1,1 \leq l \leq 2$ and $1 \leq k+l \leq 2$ in $C^{0}\left(\mathcal{C}^{\prime} \backslash\left\{\left(x_{0}^{1}, 0\right), \ldots,\left(x_{0}^{m}, 0\right)\right\}\right)$. We remind that $\mathcal{C}^{\prime}=\Omega \times[0, \infty)$ and $c_{1}=\int_{\mathbb{R}^{N}} w^{p}(x) d x>$ 0 .

Proof Take any $r>0$ small for which Lemma 4.4 holds. We are concerned with the values of $U_{n}(z)$ for $z \in A_{r}^{\prime}:=\mathcal{C}^{\prime} \backslash \cup_{i=1}^{m} \overline{B_{+}^{N+1}\left(\left(x_{0}^{i}, 0\right), r\right)}$. Let us look at

$$
U_{n}(z)=\int_{A_{r / 2}} G_{\mathcal{C}}(z, y) u_{n}^{p-\epsilon_{n}}(y) d y+\sum_{i=1}^{m} \int_{B^{N}\left(x_{0}^{i}, r / 2\right)} G_{\mathcal{C}}(z, y) u_{n}^{p-\epsilon_{n}}(y) d y .
$$

Then, by the previous lemma we have

$$
\begin{aligned}
& \left(\lambda_{n}^{1}\right)^{-\frac{N-2 s}{2}} \int_{A_{r / 2}} G_{\mathcal{C}}(z, y) u_{n}^{p-\epsilon_{n}}(y) d y \leq C\left(\lambda_{n}^{1}\right)^{-\frac{N-2 s}{2}}\left(\lambda_{n}^{m}\right)^{\frac{N-2 s}{2}\left(p-\epsilon_{n}\right)} \\
& \int_{\Omega} G_{\mathcal{C}}(z, y) d y=o(1) .
\end{aligned}
$$


Let us decompose

$$
\begin{aligned}
& \int_{B^{N}\left(x_{0}^{i}, r / 2\right)} G_{\mathcal{C}}(z, y) u_{n}^{p-\epsilon_{n}}(y) d y \\
& =G_{\mathcal{C}}\left(z, x_{0}^{i}\right) \int_{B^{N}\left(x_{0}^{i}, r / 2\right)} u_{n}^{p-\epsilon_{n}}(y) d y \\
& \quad+\int_{B^{N}\left(x_{0}^{i}, r / 2\right)}\left(G_{\mathcal{C}}(z, y)-G_{\mathcal{C}}\left(z, x_{0}^{i}\right)\right) u_{n}^{p-\epsilon_{n}}(y) d y
\end{aligned}
$$

for each $i \in\{1, \ldots, m\}$. Since

$$
\left(\lambda_{n}^{i}\right)^{\frac{N-2 s}{2}} u_{n}\left(\lambda_{n}^{i} y+x_{n}^{i}\right) \rightarrow w(y) \text { weakly in } H^{s}\left(\mathbb{R}^{N}\right),
$$

according to Corollary 3.5 and the Lebesgue dominated convergence theorem, we get

$$
\left(\lambda_{n}^{1}\right)^{-\frac{N-2 s}{2}} \int_{B^{N}\left(x_{0}^{i}, r / 2\right)} u_{n}^{p-\epsilon_{n}}(y) d y \rightarrow b_{i} \int_{\mathbb{R}^{N}} w^{p}(y) d y .
$$

Also, employing the mean value theorem, we calculate

$$
\begin{aligned}
& \left|\left(\lambda_{n}^{1}\right)^{-\frac{N-2 s}{2}} \int_{B^{N}\left(x_{0}^{i}, r / 2\right)}\left(G_{\mathcal{C}}(z, y)-G_{\mathcal{C}}\left(z, x_{0}^{i}\right)\right) u_{n}^{p-\epsilon_{n}}(y) d y\right| \\
& \leq\left(\lambda_{n}^{1}\right)^{-\frac{N-2 s}{2}} \int_{B^{N}\left(x_{0}^{i}, r / 2\right)} \sup _{z \in A_{r}^{\prime}, a \in(0,1)}\left\|\nabla_{y} G_{\mathcal{C}}\left(z, a y+(1-a) x_{0}^{i}\right)\right\| \cdot\left|y-x_{0}^{i}\right| u_{n}^{p-\epsilon_{n}}(y) d y \\
& \leq C\left(\lambda_{n}^{1}\right)^{-\frac{N-2 s}{2}} r^{1-s} \int_{B^{N}\left(x_{n}^{i}, 3 r / 4\right)}\left|y-x_{0}^{i}\right|^{s} u_{n}^{p-\epsilon_{n}}(y) d y \\
& \leq C b_{i} r^{1-s}\left[\left(\lambda_{n}^{i}\right)^{s}\left(\int_{\mathbb{R}^{N}}|y|^{s} w^{p}(y) d y+o(1)\right)+\left|x_{n}^{i}-x_{0}^{i}\right|^{s}\left(\int_{\mathbb{R}^{N}} w^{p}(y) d y+o(1)\right)\right] \\
& =o(1) .
\end{aligned}
$$

Therefore, combining all the computations, we see that (4.21) holds uniformly for $z=(x, t) \in A_{r}^{\prime}$. Since $r>0$ is arbitrary, it follows that (4.21) is valid in $C^{0}\left(\mathcal{C}^{\prime} \backslash\left\{\left(x_{0}^{1}, 0\right), \ldots,\left(x_{0}^{m}, 0\right)\right\}\right)$.

In order to show (4.22) and (4.23), we need some results on elliptic regularity. The proof is deferred to "Appendix 2."

Remark 4.7 For the future use, we rewrite (4.21) as

$$
\lim _{n \rightarrow \infty}\left(\lambda_{n}^{1}\right)^{-\frac{N-2 s}{2}} U_{n}(x, t)=\frac{c_{3} b_{i}}{\left|\left(x-x_{0}^{i}, t\right)\right|^{N-2 s}}+\mathcal{T}_{i}(x, t)
$$

for $(x, t) \in \mathcal{C}^{\prime} \backslash\left\{\left(x_{0}^{1}, 0\right), \ldots,\left(x_{0}^{m}, 0\right)\right\}$ and $1 \leq i \leq m$. Here, $c_{3}:=c_{1} \gamma_{N, s}>0$ and $\mathcal{T}_{i}$ is a map defined by

$$
\mathcal{T}_{i}(x, t)=-c_{1} b_{i} H_{\mathcal{C}}\left((x, t), x_{0}^{i}\right)+c_{1} \sum_{k \neq i} b_{k} G_{\mathcal{C}}\left((x, t), x_{0}^{k}\right) .
$$

If $r \in\left(0, d_{0} / 2\right)$ where $d_{0}>0$ is set in Lemma 4.3, then (2.9) and (2.10) imply that the functions $\mathcal{T}_{i}, \frac{\partial \mathcal{T}_{i}}{\partial x_{j}}$ and $z \cdot \nabla \mathcal{T}_{i}$ are $s$-harmonic in $B_{+}^{N+1}\left(\left(x_{0}^{i}, 0\right), r\right)$ for all $1 \leq i \leq m$ and 
$1 \leq j \leq N$, i.e.,

$$
\begin{cases}\operatorname{div}\left(t^{1-2 s} \nabla \mathcal{T}_{i}\right)=\operatorname{div}\left(t^{1-2 s} \nabla\left(\frac{\partial \mathcal{T}_{i}}{\partial x_{j}}\right)\right) & \\ =\operatorname{div}\left(t^{1-2 s} \nabla\left(z \cdot \nabla \mathcal{T}_{i}\right)\right)=0 & \text { in } B_{+}^{N+1}\left(\left(x_{0}^{i}, 0\right), r\right), \\ \partial_{v}^{s} \mathcal{T}_{i}=\partial_{v}^{s}\left(\frac{\partial \mathcal{T}_{i}}{\partial x_{j}}\right)=\partial_{v}^{s}\left(z \cdot \nabla \mathcal{T}_{i}\right)=0 & \text { on } B^{N}\left(x_{0}^{i}, r\right)\end{cases}
$$

holds.

\section{Proof of main theorems for the spectral fractional Laplacian}

This section is devoted to the proof of our main theorems. To get the desired results, we will derive two identities regarding blow-up points and rates by exploiting a type of Green's identity. For notational simplicity, we use $z-x_{0}^{i}$ to denote $\left(x-x_{0}^{i}, t\right)$ throughout the section.

As before, let $\left\{U_{n}\right\}_{n \in \mathbb{N}}$ be a sequence of solutions to (2.4) with $\epsilon=\epsilon_{n}$ of the form (4.10). We remind from (2.4) that $U_{n}$ is a solution of the problem

$$
\begin{cases}\operatorname{div}\left(t^{1-2 s} \nabla U_{n}\right)=0 & \text { in } \mathcal{C}, \\ \partial_{v}^{s} U_{n}=U_{n}^{p-\epsilon_{n}} & \text { on } \Omega \times\{0\} .\end{cases}
$$

By the translation and scaling invariance of (5.1), the functions $V=\frac{\partial U_{n}}{\partial x_{j}}$ and $V=\left(z-x_{0}^{i}\right)$. $\nabla U_{n}+\left(\frac{2 s}{p-1-\epsilon_{n}}\right) U_{n}$ (for each $1 \leq i \leq m$ and $1 \leq j \leq N$ ) satisfy the equation

$$
\begin{cases}\operatorname{div}\left(t^{1-2 s} \nabla V\right)=0 & \text { in } \mathcal{C}, \\ \partial_{v}^{s} V=\left(p-\epsilon_{n}\right) U_{n}^{p-1-\epsilon_{n}} V & \text { on } \Omega \times\{0\} .\end{cases}
$$

Lemma 5.1 Assume that a function $V \in H_{0}^{1,2}\left(\mathcal{C} ; t^{1-2 s}\right)$ satisfies (5.2). Then, for any point $y \in \Omega$, the following identity

$$
\begin{gathered}
\kappa_{s} \int_{\partial_{I} B_{+}^{N+1}((y, 0), r)} t^{1-2 s}\left(\frac{\partial U_{n}}{\partial v} V-\frac{\partial V}{\partial v} U_{n}\right) d S_{z} \\
=\left(p-1-\epsilon_{n}\right) \int_{B^{N}(y, r)} U_{n}^{p-\epsilon_{n}} V d x
\end{gathered}
$$

holds for any $r \in(0, \operatorname{dist}(y, \partial \Omega))$.

Proof Multiplying the first equation of (5.1) by $V$ and that of (5.2) by $U_{n}$, and then integrating the results over $B_{+}^{N+1}((y, 0), r)$, we obtain

$$
\begin{aligned}
& \kappa_{S} \int_{\partial_{I} B_{+}^{N+1}((y, 0), r)} t^{1-2 s}\left(\frac{\partial U_{n}}{\partial v} V-\frac{\partial V}{\partial v} U_{n}\right) d S_{z} \\
& =-\int_{B^{N}(y, r)}\left(\partial_{v}^{s} U_{n} \cdot V-\partial_{v}^{s} V \cdot U_{n}\right) \mathrm{d} x \\
& =\left(p-1-\epsilon_{n}\right) \int_{B^{N}(y, r)} U_{n}^{p-\epsilon_{n}} V \mathrm{~d} x .
\end{aligned}
$$

Here, the second equality comes from the second equations of (5.1) and (5.2). This proves (5.3). 
Based on the previous identity, we now deduce two kinds of information on the concentration points and rates.

Lemma 5.2 For any $1 \leq i \leq m$ and $1 \leq j \leq N$, we have $\frac{\partial \mathcal{T}_{i}}{\partial x_{j}}\left(x_{0}^{i}, 0\right)=0$ for $\mathcal{T}_{i}$ defined in (4.26), or equivalently,

$$
b_{i} \frac{\partial H}{\partial x_{j}}\left(x_{0}^{i}, x_{0}^{i}\right)-\sum_{k \neq i} b_{k} \frac{\partial G}{\partial x_{j}}\left(x_{0}^{i}, x_{0}^{k}\right)=0 .
$$

Proof Fix any $i \in\{1, \ldots, m\}$. Taking $V=\frac{\partial U_{n}}{\partial x_{j}}$ and $y=x_{0}^{i}$ in (5.3), we have

$$
\begin{gathered}
\kappa_{S} \int_{\partial_{I} B_{+}^{N+1}\left(\left(x_{0}^{i}, 0\right), r\right)} t^{1-2 s}\left[\frac{\partial U_{n}}{\partial v} \frac{\partial U_{n}}{\partial x_{j}}-\frac{\partial}{\partial v}\left(\frac{\partial U_{n}}{\partial x_{j}}\right) U_{n}\right] d S_{z} \\
=\left(p-1-\epsilon_{n}\right) \int_{B^{N}\left(x_{0}^{i}, r\right)} U_{n}^{p-\epsilon_{n}} \frac{\partial U_{n}}{\partial x_{j}} \mathrm{~d} x \\
=\left(\frac{p-1-\epsilon_{n}}{p+1-\epsilon_{n}}\right) \int_{\partial B^{N}\left(x_{0}^{i}, r\right)} U_{n}^{p+1-\epsilon_{n}} v_{j} d S_{x} .
\end{gathered}
$$

By lemmas 4.1, 4.4 and 4.5,

$$
\left(\lambda_{n}^{1}\right)^{-(N-2 s)}\left|\int_{\partial B^{N}\left(x_{0}^{i}, r\right)} U_{n}^{p+1-\epsilon_{n}} v_{j} d S_{x}\right|=\left(\lambda_{n}^{1}\right)^{-(N-2 s)} O\left(\left(\lambda_{n}^{i}\right)^{N-\frac{N-2 s}{2} \epsilon_{n}}\right)=o(1) .
$$

Hence, we see from (5.5) and (5.6) that

$$
\lim _{n \rightarrow \infty}\left(\lambda_{n}^{1}\right)^{-(N-2 s)} \int_{\partial_{I} B_{+}^{N+1}\left(\left(x_{0}^{i}, 0\right), r\right)} t^{1-2 s}\left[\frac{\partial U_{n}}{\partial v} \frac{\partial U_{n}}{\partial x_{j}}-\frac{\partial}{\partial v}\left(\frac{\partial U_{n}}{\partial x_{j}}\right) U_{n}\right] d S_{z}=0 .
$$

Using (4.25), we evaluate the left-hand side of (5.7) as follows:

$$
\begin{aligned}
& \lim _{n \rightarrow \infty}\left(\lambda_{n}^{1}\right)^{-(N-2 s)} \int_{\partial_{I} B_{+}^{N+1}\left(\left(x_{0}^{i}, 0\right), r\right)} t^{1-2 s}\left[\frac{\partial U_{n}}{\partial v} \frac{\partial U_{n}}{\partial x_{j}}-\frac{\partial}{\partial v}\left(\frac{\partial U_{n}}{\partial x_{j}}\right) U_{n}\right] d S_{z} \\
& =\int_{\partial_{I} B_{+}^{N+1}\left(\left(x_{0}^{i}, 0\right), r\right)} t^{1-2 s}\left(\frac{(N-2 s) c_{3} b_{i}}{\left|z-x_{0}^{i}\right|^{N-2 s+1}}-\frac{\partial \mathcal{T}_{i}}{\partial v}(z)\right) \\
& \left(\frac{(N-2 s) c_{3} b_{i}\left(x-x_{0}^{i}\right)_{j}}{\left|z-x_{0}^{i}\right|^{N-2 s+2}}-\frac{\partial \mathcal{T}_{i}}{\partial x_{j}}(z)\right) \\
& +t^{1-2 s} \frac{\partial}{\partial v}\left(\frac{(N-2 s) c_{3} b_{i}\left(x-x_{0}^{i}\right)_{j}}{\left|z-x_{0}^{i}\right|^{N-2 s+2}}-\frac{\partial \mathcal{T}_{i}}{\partial x_{j}}(z)\right) \cdot\left(\frac{c_{3} b_{i}}{\left|z-x_{0}^{i}\right|^{N-2 s}}+\mathcal{T}_{i}(z)\right) d S_{z} \\
& =\int_{\partial_{I} B_{+}^{N+1}\left(\left(x_{0}^{i}, 0\right), r\right)} t^{1-2 s}\left[-\frac{(N-2 s) c_{3} b_{i}}{\left|z-x_{0}^{i}\right|^{N-2 s+1}} \frac{\partial \mathcal{T}_{i}}{\partial x_{j}}(z)-\frac{(N-2 s) c_{3} b_{i}\left(x-x_{0}^{i}\right)_{j}}{\left|z-x_{0}^{i}\right|^{N-2 s+2}} \frac{\partial \mathcal{T}_{i}}{\partial v}(z)\right. \\
& \left.+\frac{\partial}{\partial v}\left(\frac{(N-2 s) c_{3} b_{i}\left(x-x_{0}^{i}\right)_{j}}{\left|z-x_{0}^{i}\right|^{N-2 s+2}}\right) \mathcal{T}_{i}(z)\right] d S_{z} \\
& -\int_{\partial_{I} B_{+}^{N+1}\left(\left(x_{0}^{i}, 0\right), r\right)} t^{1-2 s}\left[\frac{\partial}{\partial v}\left(\frac{\partial \mathcal{T}_{i}}{\partial x_{j}}\right) \frac{c_{3} b_{i}}{\left|z-x_{0}^{i}\right|^{N-2 s}}\right] d S_{z} \\
& +\int_{\partial_{I} B_{+}^{N+1}\left(\left(x_{0}^{i}, 0\right), r\right)} t^{1-2 s}\left[\frac{\partial \mathcal{T}_{i}}{\partial v} \frac{\partial \mathcal{T}_{i}}{\partial x_{j}}-\frac{\partial}{\partial v}\left(\frac{\partial \mathcal{T}_{i}}{\partial x_{j}}\right) \mathcal{T}_{i}\right] d S_{z}:=I_{1}+I_{2}+I_{3} .
\end{aligned}
$$


Let us compute each of the terms $I_{1}, I_{2}$ and $I_{3}$. Firstly (4.27) yields that

$$
I_{3}=-\int_{B^{N}\left(x_{0}^{i}, r\right)}\left[\partial_{v}^{s} \mathcal{T}_{i} \cdot\left(\frac{\partial \mathcal{T}_{i}}{\partial x_{j}}\right)-\partial_{v}^{s}\left(\frac{\partial \mathcal{T}_{i}}{\partial x_{j}}\right) \cdot \mathcal{T}_{i}\right] \mathrm{d} x=0 .
$$

Also, according to estimates (2.11) and (2.12), we have

$$
\begin{aligned}
\lim _{r \rightarrow 0}\left|I_{2}\right| & \leq \lim _{r \rightarrow 0} \int_{\partial_{I} B_{+}^{N+1}\left(\left(x_{0}^{i}, 0\right), r\right)} t^{1-2 s}\left|\frac{\partial}{\partial v}\left(\frac{\partial \mathcal{T}_{i}}{\partial x_{j}}\right) \frac{c_{3} b_{i}}{\left|z-x_{0}^{i}\right|^{N-2 s}}\right| d S_{z} \\
& \leq C \lim _{r \rightarrow 0} \int_{\partial_{I} B_{+}^{N+1}\left(\left(x_{0}^{i}, 0\right), r\right)} \frac{\left(t^{1-2 s}+1\right)}{\left|z-x_{0}^{i}\right|^{N-2 s}} d S_{z} \leq C \lim _{r \rightarrow 0}\left(r+r^{2 s}\right)=0 .
\end{aligned}
$$

Therefore, we only need to compute $\lim _{r \rightarrow 0} I_{1}$. By homogeneity, its first term is calculated to be

$$
\begin{aligned}
& -\lim _{r \rightarrow 0} \int_{\partial_{I} B_{+}^{N+1}\left(\left(x_{0}^{i}, 0\right), r\right)} t^{1-2 s} \frac{(N-2 s) c_{3} b_{i}}{\left|z-x_{0}^{i}\right|^{N-2 s+1}} \frac{\partial \mathcal{T}_{i}}{\partial x_{j}}(z) d S_{z} \\
& =-\frac{\partial \mathcal{T}_{i}}{\partial x_{j}}\left(x_{0}^{i}, 0\right) \cdot(N-2 s) c_{3} b_{i} \int_{\partial_{I} B_{+}^{N+1}(0,1)} \frac{t^{1-2 s}}{|z|^{N-2 s+1}} d S_{z} .
\end{aligned}
$$

For the second term, one can deduce

$$
\begin{aligned}
& -\lim _{r \rightarrow 0} \int_{\partial_{I} B_{+}^{N+1}\left(\left(x_{0}^{i}, 0\right), r\right)} t^{1-2 s} \frac{(N-2 s) c_{3} b_{i}\left(x-x_{0}^{i}\right)_{j}}{\left|z-x_{0}^{i}\right|^{N-2 s+2}} \frac{\partial \mathcal{T}_{i}}{\partial v}(z) d S_{z} \\
& =-(N-2 s) c_{3} b_{i} \cdot \lim _{r \rightarrow 0} \int_{\partial_{I} B_{+}^{N+1}\left(\left(x_{0}^{i}, 0\right), r\right)} \sum_{k=1}^{N+1} \frac{t^{1-2 s}\left(x-x_{0}^{i}\right)_{j}\left(x-x_{0}^{i}\right)_{k}}{\left|z-x_{0}^{i}\right|^{N-2 s+3}} \frac{\partial \mathcal{T}_{i}}{\partial x_{k}}(z) d S_{z} \\
& =-\frac{\partial \mathcal{T}_{i}}{\partial x_{j}}\left(x_{0}^{i}, 0\right) \cdot(N-2 s) c_{3} b_{i} \int_{\partial_{I} B_{+}^{N+1}(0,1)} \frac{t^{1-2 s} x_{j}^{2}}{|z|^{N-2 s+3}} d S_{z},
\end{aligned}
$$

because the mean value formula with (2.11) and (2.12) imply

$$
\begin{aligned}
& \left|\frac{t^{1-2 s}\left(x-x_{0}^{i}\right)_{j}\left(x-x_{0}^{i}\right)_{k}}{\left|z-x_{0}^{i}\right|^{N-2 s+3}}\left(\frac{\partial \mathcal{T}_{i}}{\partial x_{k}}(z)-\frac{\partial \mathcal{T}_{i}}{\partial x_{k}}\left(x_{0}^{i}, 0\right)\right)\right| \\
& \quad \leq C \frac{\left(1+t^{1-2 s}\right)\left|z-x_{0}^{i}\right|^{3}}{\left|z-x_{0}^{i}\right|^{N-2 s+3}}=C \frac{1+t^{1-2 s}}{\left|z-x_{0}^{i}\right|^{N-2 s}}
\end{aligned}
$$

for $1 \leq j, k \leq N+1$ so that the value of its integration over the half-sphere $\partial_{I} B_{+}^{N+1}\left(\left(x_{0}^{i}, 0\right), r\right)$ is bounded by $C\left(r+r^{2 s}\right)$ (see (5.9)). Finally, by direct computation, we discover

$$
\begin{aligned}
& \lim _{r \rightarrow 0} \int_{\partial_{I} B_{+}^{N+1}\left(\left(x_{0}^{i}, 0\right), r\right)} t^{1-2 s} \frac{\partial}{\partial v} \frac{(N-2 s) c_{3} b_{i}\left(x-x_{0}^{i}\right)_{j}}{\left|z-x_{0}^{i}\right|^{N-2 s+2}} \mathcal{T}_{i}(z) d S_{z} \\
& =-(N-2 s)(N-2 s+1) c_{3} b_{i} \lim _{r \rightarrow 0} \int_{\partial_{I} B_{+}^{N+1}\left(\left(x_{0}^{i}, 0\right), r\right)} t^{1-2 s} \frac{\left(x-x_{0}^{i}\right)_{j}}{\left|z-x_{0}^{i}\right|^{N-2 s+3}} \mathcal{T}_{i}(z) d S_{z} \\
& =-\frac{\partial \mathcal{T}_{i}}{\partial x_{j}}\left(x_{0}^{i}, 0\right) \cdot(N-2 s)(N-2 s+1) c_{3} b_{i} \int_{\partial_{I} B_{+}^{N+1}(0,1)} \frac{t^{1-2 s} x_{j}^{2}}{|z|^{N-2 s+3}} d S_{z}
\end{aligned}
$$


where we used $\mathcal{T}_{i}(x, 0)=\mathcal{T}_{i}\left(x_{0}^{i}, 0\right)+\left(x-x_{0}^{i}\right) \cdot \nabla_{x} \mathcal{T}_{i}\left(x_{0}^{i}, 0\right)+O\left(\left|x-x_{0}^{i}\right|^{2}\right)$ to find the second equality. Thus (5.7) is reduced to

$$
\begin{aligned}
& -\frac{\partial \mathcal{T}_{i}}{\partial x_{j}}\left(x_{0}^{i}, 0\right) \cdot\left(\int_{\partial_{I} B_{+}^{N+1}(0,1)} \frac{t^{1-2 s}}{|z|^{N-2 s+1}} d S_{z}\right. \\
& \left.\quad+(N-2 s+2) \int_{\partial_{I} B_{+}^{N+1}(0,1)} \frac{t^{1-2 s} x_{j}^{2}}{|z|^{N-2 s+3}} d S_{z}\right)=0 .
\end{aligned}
$$

Therefore, $\frac{\partial \mathcal{T}_{i}}{\partial x_{j}}\left(x_{0}^{i}, 0\right)=0$, proving the lemma.

Remark 5.3 It is shown in [25, Section 4] that

$$
\int_{\partial_{I} B_{+}^{N+1}(0,1)} \frac{t^{1-2 s}}{|z|^{N-2 s+1}} d S_{z}=\frac{\left|S^{N-1}\right|}{2} B\left(1-s, \frac{N}{2}\right)
$$

and

$$
\begin{aligned}
\int_{\partial_{I} B_{+}^{N+1}(0,1)} \frac{t^{1-2 s} x_{1}^{2}}{|z|^{N-2 s+3}} d S_{z} & =\frac{\left|S^{N-1}\right|}{2 N} B\left(1-s, \frac{N+2}{2}\right) \\
& =\frac{1}{N-2 s+2} \int_{\partial_{I} B_{+}^{N+1}(0,1)} \frac{t^{1-2 s}}{|z|^{N-2 s+1}} d S_{z}
\end{aligned}
$$

where $B$ is the Beta function.

Lemma 5.4 For each $1 \leq i \leq m$, we have

$$
b_{i}^{2} H\left(x_{0}^{i}, x_{0}^{i}\right)-\sum_{k \neq i} b_{i} b_{k} G\left(x_{0}^{i}, x_{0}^{k}\right)=\frac{c_{2}}{2 c_{1}} b_{0}
$$

where $c_{2}>0$ in (1.7) and $b_{0}=\lim _{n \rightarrow \infty}\left(\lambda_{n}^{1}\right)^{-(N-2 s)} \epsilon_{n}$.

Proof Fix $i \in\{1, \ldots, m\}$. Taking $V=V_{n}=\left(z-x_{0}^{i}\right) \cdot \nabla U_{n}+\left(\frac{2 s}{p-1-\epsilon_{n}}\right) U_{n}$ and $y=x_{0}^{i}$ in (5.3), we find

$$
\begin{aligned}
& \kappa_{s} \lim _{n \rightarrow \infty}\left(\lambda_{n}^{1}\right)^{-(N-2 s)} \int_{\partial_{I} B_{+}^{N+1}\left(\left(x_{0}^{i}, 0\right), r\right)} t^{1-2 s}\left[\frac{\partial U_{n}}{\partial v} V_{n}-\frac{\partial V_{n}}{\partial v} U_{n}\right] d S_{z} \\
& =\lim _{n \rightarrow \infty}\left(\lambda_{n}^{1}\right)^{-(N-2 s)}\left(p-1-\epsilon_{n}\right) \int_{B^{N}\left(x_{0}^{i}, r\right)} u_{n}^{p-\epsilon_{n}} v_{n} \mathrm{~d} x
\end{aligned}
$$

where $v_{n}=\left.\operatorname{tr}\right|_{\Omega \times\{0\}} V_{n}$. To evaluate the left-hand side of (5.12), we observe from (4.25) that

$$
\begin{aligned}
\lim _{n \rightarrow \infty}\left(\lambda_{n}^{1}\right)^{-\frac{N-2 s}{2}} V_{n}(z)=- & \left(\frac{N-2 s}{2}\right) \frac{c_{3} b_{i}}{\left|z-x_{0}^{i}\right|^{N-2 s}} \\
& +\left(z-x_{0}^{i}\right) \cdot \nabla \mathcal{T}_{i}(z)+\left(\frac{N-2 s}{2}\right) \mathcal{T}_{i}(z)
\end{aligned}
$$


for $z=(x, t) \in \mathcal{C}^{\prime} \backslash\left\{\left(x_{0}^{1}, 0\right), \ldots,\left(x_{0}^{m}, 0\right)\right\}$. Thus, we get

$$
\begin{aligned}
& \lim _{n \rightarrow \infty}\left(\lambda_{n}^{1}\right)^{-(N-2 s)} \int_{\partial_{I} B_{+}^{N+1}\left(\left(x_{0}^{i}, 0\right), r\right)} t^{1-2 s}\left[\frac{\partial U_{n}}{\partial v} V_{n}-\frac{\partial V_{n}}{\partial v} U_{n}\right] d S_{z} \\
&=-\int_{\partial_{I} B_{+}^{N+1}\left(\left(x_{0}^{i}, 0\right), r\right)} t^{1-2 s} \frac{(N-2 s) c_{3} b_{i}}{\left|z-x_{0}^{i}\right|^{N-2 s+1}}\left(\left(z-x_{0}^{i}\right) \cdot \nabla \mathcal{T}_{i}+(N-2 s) \mathcal{T}_{i}\right) d S_{z} \\
& \quad-\int_{\partial_{I} B_{+}^{N+1}\left(\left(x_{0}^{i}, 0\right), r\right)} t^{1-2 s} \frac{c_{3} b_{i}}{\left|z-x_{0}^{i}\right|^{N-2 s}} \frac{\partial}{\partial v}\left(\left(z-x_{0}^{i}\right) \cdot \nabla \mathcal{T}_{i}+(N-2 s) \mathcal{T}_{i}\right) d S_{z} \\
&+\int_{\partial_{I} B_{+}^{N+1}\left(\left(x_{0}^{i}, 0\right), r\right)} t^{1-2 s}\left[\frac{\partial \mathcal{T}_{i}}{\partial v}\left(\left(z-x_{0}^{i}\right) \cdot \nabla \mathcal{T}_{i}+\left(\frac{N-2 s}{2}\right) \mathcal{T}_{i}\right)\right. \\
&\left.-\mathcal{T}_{i} \frac{\partial}{\partial v}\left(\left(z-x_{0}^{i}\right) \cdot \nabla \mathcal{T}_{i}+\left(\frac{N-2 s}{2}\right) \mathcal{T}_{i}\right)\right] d S_{z} \\
&:= J_{1}+J_{2}+J_{3} .
\end{aligned}
$$

As the previous proof, let us estimate each of $J_{1}, J_{2}$ and $J_{3}$. As demonstrated in (5.8), we have $J_{3}=0$. Besides (2.11) and (2.12) lead us to derive

$$
\lim _{r \rightarrow 0}\left|J_{2}\right| \leq C \lim _{r \rightarrow 0} \int_{\partial_{I} B_{+}^{N+1}\left(\left(x_{0}^{i}, 0\right), r\right)} \frac{\left(t^{1-2 s}+1\right)}{\left|z-x_{0}^{i}\right|^{N-2 s}} d S_{z}=0 .
$$

Lastly, since

$$
\left.\left[\left(z-x_{0}^{i}\right) \cdot \nabla \mathcal{T}_{i}(z)+(N-2 s) \mathcal{T}_{i}(z)\right]\right|_{z=\left(x_{0}^{i}, 0\right)}=(N-2 s) \mathcal{T}_{i}\left(x_{0}^{i}, 0\right),
$$

we have

$$
\lim _{r \rightarrow 0} J_{1}=-c_{3} b_{i}(N-2 s)^{2}\left(\int_{\partial_{I} B_{+}^{N+1}(0,1)} \frac{t^{1-2 s}}{|z|^{N-2 s+1}} d S_{z}\right) \mathcal{T}_{i}\left(x_{0}^{i}, 0\right) .
$$

As a result, after the limit $r \rightarrow 0$ being taken, the left-hand side of (5.12) becomes

$$
\begin{aligned}
& c_{1} c_{3} \kappa_{S}(N-2 s)^{2}\left(\int_{\partial_{I} B_{+}^{N+1}(0,1)} \frac{t^{1-2 s}}{|z|^{N-2 s+1}} d S_{z}\right)\left[b_{i}^{2} H_{\mathcal{C}}\left(\left(x_{0}^{i}, 0\right), x_{0}^{i}\right)\right. \\
& \left.-\sum_{k \neq i} b_{i} b_{k} G_{\mathcal{C}}\left(\left(x_{0}^{i}, 0\right), x_{0}^{k}\right)\right] .
\end{aligned}
$$

Meanwhile, using integration by parts, we deduce that

$$
\begin{aligned}
& \int_{B^{N}\left(x_{i}^{0}, r\right)} u_{n}^{p-\epsilon_{n}}\left[\left(x-x_{0}^{i}\right) \cdot \nabla_{x} u_{n}+\left(\frac{2 s}{p-1-\epsilon_{n}}\right) u_{n}\right] \mathrm{d} x \\
& =\frac{1}{p+1-\epsilon_{n}} \int_{B^{N}\left(x_{i}^{0}, r\right)}\left(x-x_{0}^{i}\right) \cdot \nabla_{x} u_{n}^{p+1-\epsilon_{n}} \mathrm{~d} x+\frac{2 s}{p-1-\epsilon_{n}} \int_{B^{N}\left(x_{i}^{0}, r\right)} u_{n}^{p+1-\epsilon_{n}} \mathrm{~d} x \\
& =\frac{1}{p+1-\epsilon_{n}} \int_{\partial B^{N}\left(x_{i}^{0}, r\right)}\left(x-x_{0}^{i}\right) \cdot v u_{n}^{p+1-\epsilon_{n}} d S_{x} \\
& \quad+\left(\frac{2 s}{p-1-\epsilon_{n}}-\frac{N}{p+1-\epsilon_{n}}\right) \int_{B^{N}\left(x_{i}^{0}, r\right)} u_{n}^{p+1-\epsilon_{n}} \mathrm{~d} x .
\end{aligned}
$$


Note that

$$
\frac{2 s}{p-1-\epsilon_{n}}-\frac{N}{p+1-\epsilon_{n}}=\frac{(N-2 s) \epsilon_{n}}{\left(\frac{4 s}{N-2 s}-\epsilon_{n}\right)\left(\frac{2 N}{N-2 s}-\epsilon_{n}\right)}=\frac{(N-2 s)^{3} \epsilon_{n}}{8 N s}(1+o(1))
$$

and

$$
\int_{\partial B^{N}\left(x_{i}^{0}, r\right)}\left(x-x_{0}^{i}\right) \cdot v u_{n}^{p+1-\epsilon_{n}} d S_{x}=O\left(\left(\lambda_{n}^{1}\right)^{N}\right) .
$$

Hence, the right-hand side of (5.12) equals to

$$
\left(\lambda_{n}^{1}\right)^{-(N-2 s)} \epsilon_{n}(1+o(1)) \cdot \frac{(N-2 s)^{2}}{2 N} \int_{\mathbb{R}^{N}} w^{p+1} \mathrm{~d} x+O\left(\left(\lambda_{n}^{1}\right)^{2 s}\right) .
$$

From (5.12), (5.13), (5.14) and (5.10), we get

$$
\begin{aligned}
\frac{b_{0}}{N} \int_{\mathbb{R}^{N}} w^{p+1} \mathrm{~d} x= & c_{1} c_{3} \kappa_{s}\left|S^{N-1}\right| B\left(1-s, \frac{N}{2}\right) \\
& \times\left[b_{i}^{2} H_{\mathcal{C}}\left(\left(x_{0}^{i}, 0\right), x_{0}^{i}\right)-\sum_{k \neq i} b_{i} b_{k} G_{\mathcal{C}}\left(\left(x_{0}^{i}, 0\right), x_{0}^{k}\right)\right] \\
= & \frac{2}{N-2 s}\left(\int_{\mathbb{R}^{N}} w^{p} \mathrm{~d} x\right)^{2}\left[b_{i}^{2} H\left(x_{0}^{i}, x_{0}^{i}\right)-\sum_{k \neq i} b_{i} b_{k} G\left(x_{0}^{i}, x_{0}^{k}\right)\right] .
\end{aligned}
$$

This completes the proof.

We are now prepared to complete the proof of our main theorems.

Proof of Theorem 1.1 Assume that $\sup _{n \in \mathbb{N}}\left\|u_{n}\right\|_{\widetilde{H}^{s}(\Omega)}<\infty$. Then, if we let $U_{n}$ be the $s$-harmonic extension of $u_{n}$ over the half-cylinder $\mathcal{C}=\Omega \times(0, \infty)$, we have $\sup _{n \in \mathbb{N}}\left\|U_{n}\right\|_{H_{0}^{1,2}\left(\mathcal{C} ; t^{1-2 s}\right)}<\infty$ by inequality (2.5). Thus, we can apply Lemma 2.2 to the sequence $\left\{U_{n}\right\}_{n \in \mathbb{N}}$ to deduce the existence of an integer $m \in \mathbb{N} \cup\{0\}$ and sequences of positive numbers and points $\left\{\left(\lambda_{n}^{i}, x_{n}^{i}\right)\right\}_{n \in \mathbb{N}} \subset(0, \infty) \times \Omega$ for each $i=1, \ldots, m$ such that relation (2.21) holds (in particular $\lambda_{n}^{i} \rightarrow 0$ ) and

$$
U_{n}-\left(V_{0}+\sum_{i=1}^{m} P W_{\lambda_{n}^{i}, x_{n}^{i}}\right) \rightarrow 0 \text { in } H_{0}^{1,2}\left(\mathcal{C} ; t^{1-2 s}\right) \text { as } n \rightarrow \infty
$$

along a subsequence. Here, $V_{0}$ is the weak limit of $U_{n}$ in $H_{0}^{1,2}\left(\mathcal{C} ; t^{1-2 s}\right)$, which is a solution to (2.20), and $P W_{\lambda_{n}^{i}, x_{n}^{i}}$ is the projected bubble whose definition can be found in (2.17).

We now split the problem into two cases.

Case $1(m=0)$. By (2.2) and the strong maximum principle, $v_{0}(x)=V_{0}(x, 0)$ for $x \in \Omega$ satisfies Eq. (1.4). In addition, by (5.15), it holds that

$$
\lim _{n \rightarrow \infty}\left\|u_{n}-v\right\|_{\widetilde{H}^{s}(\Omega)}=\lim _{n \rightarrow \infty}\left\|U_{n}-V_{0}\right\|_{H_{0}^{1,2}\left(\mathcal{C} ; t^{1-2 s}\right)}=0 .
$$

This case corresponds to the first alternative (1) of Theorem 1.1. 
Case $2(m \geq 1)$. Thanks to Lemma 4.2, we have $V_{0}=0$ in this situation. Hence (5.15) and discussion in Sect. 2.5 give decomposition (1.5) as well as $x_{n}^{i} \rightarrow x_{0}^{i} \in \Omega$. Also, by lemmas 4.3 and 4.5 , there are constants $d_{0}, C_{0}>0$ independent of $n \in \mathbb{N}$ such that

$$
\left|x_{0}^{i}-x_{0}^{j}\right| \geq d_{0} \text { and } \frac{\lambda_{n}^{i}}{\lambda_{n}^{j}} \leq C_{0} \text { for any } 1 \leq i \neq j \leq m \text {. }
$$

Thus, we may set a positive value $b_{i}=\lim _{n \rightarrow \infty}\left(\frac{\lambda_{n}^{i}}{\lambda_{n}^{1}}\right)^{\frac{N-2 s}{2}}$ for each $1 \leq i \leq m$. Furthermore, lemmas 5.2 and 5.4 imply that $\left(\left(b_{1}, \ldots, b_{m}\right),\left(x_{0}^{1}, \ldots, x_{0}^{m}\right)\right) \subset(0, \infty)^{m} \times$ $\Omega^{m}$ is a critical point of the function $\Phi_{m}$ introduced in (1.6). We have proved that the case $m \geq 1$ corresponds to the second alternative (2) in Theorem 1.1. The proof is finished.

Proof of Theorem 1.3 The fact that $M$ is a nonnegative matrix can be shown as in Appendix A of [5], so we left it to the reader.

Suppose that $M$ is nondegenerate. Since the left-hand side of (5.11) is finite, it should hold that $b_{0} \in[0, \infty)$. To the contrary, let us assume that $b_{0}=0$. Then, we see

$$
b_{i} H\left(x_{0}^{i}, x_{0}^{i}\right)-\sum_{k \neq i} b_{j} G\left(x_{0}^{i}, x_{0}^{k}\right)=0
$$

for each $1 \leq i \leq m$. It means that $\mathbf{b}=\left(b_{1}, \ldots, b_{m}\right)$ is a nonzero vector such that $M \mathbf{b}=0$. However, this is nonsense because the nondegeneracy condition of $M$ tells us that $\mathbf{b}=0$. Hence, $b_{0} \neq 0$ should be true, and thus

$$
\lim _{n \rightarrow \infty} \log _{\epsilon_{n}} \lambda_{n}^{i}=\lim _{n \rightarrow \infty} \log _{\epsilon_{n}}\left[\epsilon_{n}^{\frac{1}{N-2 s}}\left(b_{0}^{-\frac{1}{N-2 s}}+o(1)\right)\left(b_{i}^{\frac{2}{N-2 s}}+o(1)\right)\right]=\frac{1}{N-2 s} .
$$

The proof is now complete.

\section{The restricted fractional Laplacian and the classical Laplacian}

\subsection{Proof of Theorems 1.1 and 1.3 for the restricted fractional Laplacian}

Here, we briefly mention how the proof for the main theorems 1.1 and 1.3 can be carried out for the restricted fractional Laplacian.

First of all, as mentioned before, the Struwe's concentration-compactness principle-type result (Step 1 in Introduction) can be obtained as in [2,31,48]. Besides the moving plane argument in Sect. 3 (corresponding to Step 2) is local in nature, so the same proof as in Sect. 3 works. For Sect. 4, one can check each lemma remains valid even if (2.4) is replaced with (2.6). Finally, we notice that lemmas 5.2 and 5.4 were obtained from the information on the solutions $\left\{U_{n}\right\}_{n \in \mathbb{N}}$ to (2.4) over the half-balls $\left\{B_{+}^{N+1}\left(\left(x_{0}^{i}, 0\right), r\right)\right\}_{i=1}^{m}$. Therefore, the same argument goes through for (2.6), completing Step 3. Theorems 1.1 and 1.3 for the restricted fractional Laplacians now follow.

\subsection{Proof of Theorem B}

To validate Theorem B, we follow the strategy used to prove Theorems 1.1 and 1.3 for nonlocal problems. 
The representation formula (1.10) of finite energy solutions $\left\{u_{n}\right\}_{n \in \mathbb{N}}$ to (1.9) is due to Struwe [59] (Step 1). Also, as in [26, Appendix A], a moving sphere argument can be applied to deduce a pointwise upper bound of $u_{n}$. It implies lemmas 4.2, 4.3 and 4.5 for the local case, which are originally given in [55]. It can be easily seen that Lemma 4.1 remains true, and the local versions of lemmas 4.4 and 4.6 are found in [26, Section 2], whence Step 2 is finished. Regarding Lemma 5.3, we have

Lemma 6.1 Suppose that a function $v \in H_{0}^{1,2}(\Omega)$ satisfies

$$
-\Delta v=\left(p-\epsilon_{n}\right) u_{n}^{p-1-\epsilon_{n}} v \text { in } \Omega \text {. }
$$

Then, for any point $y \in \Omega$, the following identity

$$
\int_{\partial B^{N}(y, r)}\left(\frac{\partial u}{\partial v} v-\frac{\partial v}{\partial v} u\right) d S_{x}=\left(p-1-\epsilon_{n}\right) \int_{B^{N}(y, r)} u_{n}^{p-\epsilon_{n}} v d x
$$

holds for any $r \in(0, \operatorname{dist}(y, \partial \Omega))$.

By taking $u=u_{n}$ and $v=\frac{\partial u_{n}}{\partial x_{j}}$ for $j=1, \ldots, N$ or $v=\left(x-x_{0}^{i}\right) \cdot \nabla u_{n}+\left(\frac{2}{p-1-\epsilon_{n}}\right) u_{n}$ for $i=1, \ldots, m$ in (6.1), we get lemmas 5.2 and 5.4 where the constants $c_{1}$ and $c_{2}$ are given by (1.7) with $s=1$. Thus, Step 3 is done. Putting all the results together, we complete the proof of Theorem B.

Acknowledgements W. Choi is grateful to the financial support from POSCO TJ Park Foundation. S. Kim is supported by FONDECYT Grant 3140530. The authors wish to thank the anonymous referee for pointing out and correcting several inaccuracies occurred in selecting appropriate functional spaces and norms to work with.

\section{Appendix 1: Lower and upper estimates of the standard bubble in $\mathbb{R}_{+}^{N+1}$}

Here, we shall prove a decay estimate of $W_{\lambda, 0}$, which is necessary in applying the moving sphere argument (see Sect. 3).

Lemma 7.1 For any $\eta>0$ there exists $R=R(\eta)>1$ so large that

$$
\alpha_{N, s}(1-\eta) \lambda^{\frac{N-2 s}{2}}|z|^{-(N-2 s)} \leq W_{\lambda, 0}(z) \leq \alpha_{N, s}(1+\eta) \lambda^{\frac{N-2 s}{2}}|z|^{-(N-2 s)} \quad \text { for all }|z|>R
$$

where $\alpha_{N, s}>0$ is the constant defined in Notations.

Proof Since $W_{\lambda, 0}(z)=\lambda^{-\frac{N-2 s}{2}} W\left(\lambda^{-1} z\right)$, we may assume that $\lambda=1$. Let us prove the lower estimate first. Taking a small number $\delta>0$ to be determined later, we consider two exclusive cases: (1) $|x|>\delta|t|$ and (2) $|x| \leq \delta|t|$. 
For the case (1), we see from Green's representation formula, (2.8) and (2.13) that

$$
\begin{aligned}
W(x, t) & \geq \alpha_{N, s}^{p} \gamma_{N, s} \int_{|y| \leq \delta|x|} \frac{1}{|(x-y, t)|^{N-2 s}} \frac{1}{\left(1+|y|^{2}\right)^{\frac{N+2 s}{2}}} d y \\
& \geq \frac{1}{|((1+\delta) x, t)|^{N-2 s}} \cdot \alpha_{N, s}^{p} \gamma_{N, s} \int_{|y| \leq \delta|x|} \frac{1}{\left(1+|y|^{2}\right)^{\frac{N+2 s}{2}}} d y \\
& \geq \frac{1}{(1+\delta)^{N-2 s}|(x, t)|^{N-2 s}}\left(\alpha_{N, s}^{p} \gamma_{N, s} \int_{\mathbb{R}^{N}} \frac{1}{\left(1+|y|^{2}\right)^{\frac{N+2 s}{2}}} d y-o(1)\right) \\
& =\frac{1}{(1+\delta)^{N-2 s}|(x, t)|^{N-2 s}}\left(\alpha_{N, s}-o(1)\right)
\end{aligned}
$$

where $o(1) \rightarrow 0$ as $|z|=|(x, t)| \rightarrow \infty$.

For the case (2), we have

$$
\begin{aligned}
W(x, t) & \geq \alpha_{N, s}^{p} \gamma_{N, s} \int_{|y| \leq \delta|t|} \frac{1}{|(x-y, t)|^{N-2 s}} \frac{1}{\left(1+|y|^{2}\right)^{\frac{N+2 s}{2}}} d y \\
& \geq \frac{1}{(1+2 \delta)^{N-2 s}|t|^{N-2 s}} \cdot \alpha_{N, s}^{p} \gamma_{N, s} \int_{|y| \leq \delta|t|} \frac{1}{\left(1+|y|^{2}\right)^{\frac{N+2 s}{2}}} d y \\
& \geq \frac{1}{(1+2 \delta)^{N-2 s}|(x, t)|^{N-2 s}}\left(\alpha_{N, s}-o(1)\right)
\end{aligned}
$$

where $o(1) \rightarrow 0$ as $|z|=|(x, t)| \rightarrow \infty$.

Hence, if we choose $\delta>0$ small and $R>0$ large so that

$$
\frac{1}{(1+2 \delta)^{N-2 s}} \geq 1-\frac{\eta}{2} \text { and } \alpha_{N, s}-o(1) \geq\left(1-\frac{\eta}{2}\right) \alpha_{N, s},
$$

we obtain the desired estimate from (7.2) and (7.3).

We turn to prove the upper estimate. Again, we take into account the cases (1) $|x|>\delta|t|$ and (2) $|x| \leq \delta|t|$ separately.

For the case (1), we estimate

$$
\begin{aligned}
& \alpha_{N, s}^{p} \gamma_{N, s} \int_{|y| \leq \delta|x|} \frac{1}{|(x-y, t)|^{N-2 s}} \frac{1}{\left(1+|y|^{2}\right)^{\frac{N+2 s}{2}}} d y \leq \frac{\alpha_{N, s}}{|((1-\delta) x, t)|^{N-2 s}} \\
& \quad \leq \frac{1}{(1-\delta)^{N-2 s}} \frac{\alpha_{N, s}}{|(x, t)|^{N-2 s}}
\end{aligned}
$$

and

$$
\begin{aligned}
& \alpha_{N, s}^{p} \gamma_{N, s} \int_{|y| \geq \delta|x|} \frac{1}{|(x-y, t)|^{N-2 s}} \frac{1}{\left(1+|y|^{2}\right)^{\frac{N+2 s}{2}}} d y \\
& =\alpha_{N, s}^{p} \gamma_{N, s}\left(\int_{2|x| \geq|y| \geq \delta|x|}+\int_{|y| \geq 2|x|}\right) \frac{1}{|(x-y, t)|^{N-2 s}} \frac{1}{\left(1+|y|^{2}\right)^{\frac{N+2 s}{2}}} d y \\
& \leq \alpha_{N, s}^{p} \gamma_{N, s}\left(\int_{2|x| \geq|y| \geq \delta|x|} \frac{1}{|x-y|^{N-2 s}} \frac{1}{(\delta|x|)^{N+2 s}} d y\right. \\
& \left.\quad+\int_{|y| \geq 2|x|} \frac{1}{|x|^{N-2 s}} \frac{1}{\mid\left(1+|y|^{2}\right)^{\frac{N+2 s}{2}}} d y\right) \\
& \leq \frac{\alpha_{N, s}^{\prime}}{\delta^{N+2 s}|x|^{N}} \leq \frac{2^{N / 2} \alpha_{N, s}^{\prime}}{\delta^{2(N+s)}|(x, t)|^{N}}
\end{aligned}
$$


where $\alpha_{N, s}^{\prime}>0$ is a certain constant relying only on $N$ and $s$. Observe that the last inequality came from $|(x, t)|<\sqrt{1+\delta^{-2}}|x| \leq \sqrt{2} \delta^{-1}|x|$ for $\delta>0$ small enough. Combining the above estimates, we get

$$
W(x, t) \leq \frac{1}{(1-\delta)^{N-2 s}} \frac{\alpha_{N, s}}{|(x, t)|^{N-2 s}}+\frac{2^{N / 2} \alpha_{N, s}^{\prime}}{\delta^{2(N+s)}|(x, t)|^{N}} .
$$

For the case (2), we have

$$
\begin{aligned}
W(x, t) & \leq \alpha_{N, s}^{p} \gamma_{N, s} \int_{\mathbb{R}^{N}} \frac{1}{|t|^{N-2 s}} \frac{1}{\left(1+|y|^{2}\right)^{\frac{N+2 s}{2}}} d y=\frac{\alpha_{N, s}}{|t|^{N-2 s}} \\
& \leq(1+\delta)^{N-2 s} \frac{\alpha_{N, s}}{|(x, t)|^{N-2 s}} .
\end{aligned}
$$

Consequently, with the choices

$$
\frac{1}{(1-\delta)^{N-2 s}} \leq 1+\frac{\eta}{2} \text { and } \frac{2^{N / 2} \alpha_{N, s}^{\prime}}{\delta^{2(N+s)} R^{N}} \leq \frac{\eta}{2},
$$

estimates (7.4) and (7.5) imply the second inequality of the lemma. The proof is completed.

\section{Appendix 2: Elliptic regularity results and derivation of (4.22) and (4.23)}

This section is devoted to present some elliptic regularity results and its application to justification of (4.22) and (4.23). For brevity, we denote

$Q_{r}=B_{+}^{N+1}((x, 0), r)$ and $B_{r}=B^{N}(x, r)$ for any fixed $x \in \Omega, 0<r<\operatorname{dist}(x, \partial \Omega) / 2$. Also, $\partial_{i}=\partial_{x_{i}}$ for $1 \leq i \leq N$.

We need to recall two lemmas which can be proved with the Moser iteration method. One is an a priori $L^{\infty}$-estimate. See, for example, [25, Lemma 3.8], [34, Theorem 3.4] and [39, propositions $2.3,2.6]$.

Lemma 8.1 Let $U \in H_{0}^{1,2}\left(Q_{2 r} ; t^{1-2 s}\right)$ be a weak solution to

$$
\begin{cases}\operatorname{div}\left(t^{1-2 s} \nabla U\right)=0 & \text { in } Q_{2 r}, \\ \partial_{\nu}^{s} U=a U+f & \text { on } B_{2 r}\end{cases}
$$

and assume that $\|a\|_{L^{\frac{N}{2 s}}\left(B_{2 r}\right)}<\delta$ for a small value $\delta=\delta(N, s)>0$. If $f \in L^{q}\left(B_{r}\right)$ for some $q>\frac{n}{2 s}$ and $\theta \in(0,1)$, then, we have

$$
\|U\|_{L^{\infty}\left(Q_{\theta r}\right)}^{2}+\int_{Q_{\theta r}} t^{1-2 s}|\nabla U|^{2} d z \leq C\left(\int_{Q_{r}} t^{1-2 s}|U|^{2} d z+\|f\|_{L^{q}\left(B_{r}\right)}^{2}\right)
$$

for some $C=C(N, s, r, \theta)>0$.

The other is a result on Hölder estimates. Refer to [39, Proposition 2.6] and [12, Lemma 4.5].

Lemma 8.2 Let $U \in H_{0}^{1,2}\left(Q_{2 r} ; t^{1-2 s}\right)$ be a weak solution to

$$
\begin{cases}\operatorname{div}\left(t^{1-2 s} \nabla U\right)=0 & \text { in } Q_{2 r}, \\ \partial_{v}^{s} U=f & \text { on } B_{2 r},\end{cases}
$$


and $\theta \in(0,1)$.

(1) If $f \in L^{q}\left(B_{r}\right)$ for some $q>\frac{N}{2 s}$, then for some $\alpha \in(0,1)$, we have

$$
\|U\|_{C^{\alpha}\left(Q_{\theta r}\right)} \leq C\left(\|U\|_{L^{\infty}\left(Q_{r}\right)}+\|f\|_{L^{q}\left(B_{r}\right)}\right) .
$$

(2) If $f \in C^{\beta}\left(B_{r}\right)$ for some $\beta \in(0,1)$, then there exists $\alpha \in(0,1)$ such that

$$
\left\|t^{1-2 s} \partial_{t} U\right\|_{C^{\alpha}\left(Q_{\theta r}\right)} \leq C\left(\|U\|_{L^{\infty}\left(Q_{r}\right)}+\|f\|_{C^{\beta}\left(B_{r}\right)}\right) .
$$

Now, we are ready to prove the main result of this section.

Proposition 8.3 Let $1<q \leq \frac{N+2 s}{N-2 s}$. Suppose that $U \in H_{0}^{1,2}\left(Q_{2 r} ; t^{1-2 s}\right)$ is a positive solution of

$$
\begin{cases}\operatorname{div}\left(t^{1-2 s} \nabla U\right)=0 & \text { in } Q_{2 r}, \\ \partial_{v}^{s} U=U^{q} & \text { on } B_{2 r} .\end{cases}
$$

Assume that $\int_{B_{2 r}} U^{\frac{N}{2 s}}(q-1)^{2}(x, 0) d x \leq \delta$ for some small value $\delta=\delta(N, s)>0$. Then, $U(x, t)$ is twice differentiable in the $x$-variable in $Q_{r / 2}$. Moreover, the following estimates hold:

$$
\begin{aligned}
&\left\|\nabla_{x} U\right\|_{C^{\alpha}\left(Q_{r / 2}\right)} \leq C\left(1+\left\|U^{q-1}\right\|_{L^{\infty}\left(B_{r}\right)}\right)\|U\|_{L^{\infty}\left(Q_{r}\right)}, \\
&\left\|t^{1-2 s} \partial_{t} U\right\|_{C^{\alpha}\left(Q_{r / 2}\right)} \leq C\left(\|U\|_{L^{\infty}\left(Q_{r}\right)}+\left\|U^{q}\right\|_{C^{1}\left(B_{r}\right)}\right), \\
&\left\|\nabla_{x}^{2} U\right\|_{C^{\alpha}\left(Q_{r / 2}\right)} \leq C\left(1+\left\|U^{q-1}\right\|_{L^{\infty}\left(B_{r}\right)}\right)\left(\|U\|_{L^{\infty}\left(Q_{r}\right)}+\left\|U^{q-2}\left|\nabla_{x} U\right|^{2}\right\|_{L^{\infty}\left(B_{r}\right)}\right), \\
&\left\|t^{1-2 s} \partial_{t} \nabla_{x} U\right\|_{C^{\alpha}\left(Q_{r / 2}\right)} \leq C\left(\left\|\nabla_{x} U\right\|_{L^{\infty}\left(Q_{r}\right)}+\left\|U^{q-1}\left|\nabla_{x} U\right|\right\|_{C^{1}\left(B_{r}\right)}\right), \\
&\left\|t^{2-2 s} \partial_{t}^{2} U\right\|_{C^{\alpha}\left(Q_{r / 2}\right)} \leq C\left(\left\|t^{1-2 s} \partial_{t} U\right\|_{C^{\alpha}\left(Q_{r / 2}\right)}+\left\|t^{2-2 s}\left|\nabla_{x}^{2} U\right|\right\|_{C^{\alpha}\left(Q_{r / 2}\right)}\right)
\end{aligned}
$$

for some $\alpha \in(0,1)$.

Proof By Propositions 2.13 and 2.19 of [39], any positive solution $U$ to (8.1) is twice differentiable in $x$ and it holds that

$$
\begin{cases}\operatorname{div}\left(t^{1-2 s} \nabla \partial_{i} U\right)=0 & \text { in } Q_{r} \\ \partial_{v}^{s}\left(\partial_{i} U\right)=q U^{q-1} \partial_{i} U & \text { on } B_{r}\end{cases}
$$

and

$$
\begin{cases}\operatorname{div}\left(t^{1-2 s} \nabla \partial_{i} \partial_{j} U\right)=0 & \text { in } Q_{r}, \\ \partial_{v}^{s}\left(\partial_{i} \partial_{j} U\right)=q U^{q-1} \partial_{i} \partial_{j} U+q(q-1) U^{q-2}\left(\partial_{i} U\right)\left(\partial_{j} U\right) & \text { on } B_{r}\end{cases}
$$

for any $1 \leq i, j \leq N$.

Let us prove validity of the estimates. Applying Lemma 8.1 to equations (8.1) and (8.2), we get

$$
\begin{aligned}
\int_{Q_{4 r / 5}} t^{1-2 s}\left|\nabla \partial_{i} U\right|^{2} d z & \leq C \int_{Q_{5 r / 6}} t^{1-2 s}\left|\partial_{i} U\right|^{2} d z \\
& \leq C \int_{Q_{r}} t^{1-2 s}|U|^{2} d z \leq C\|U\|_{L^{\infty}\left(Q_{r}\right)}^{2} .
\end{aligned}
$$

Using this chain of inequalities and Lemma 8.1 once more, we find

$$
\begin{aligned}
\left\|\partial_{i} U\right\|_{L^{\infty}\left(Q_{3 r / 4}\right)}^{2} & \leq C \int_{Q_{4 r / 5}} t^{1-2 s}\left|\partial_{i} U\right|^{2} d z \\
& \leq C\|U\|_{L^{\infty}\left(Q_{r}\right)}^{2} .
\end{aligned}
$$


Hence, Lemma 8.2 (1) gives the first inequality of Proposition 8.3

$$
\begin{aligned}
\left\|\partial_{i} U\right\|_{C^{\alpha}\left(Q_{r / 2}\right)} & \leq C\left(\left\|\partial_{i} U\right\|_{L^{\infty}\left(Q_{3 r / 4}\right)}+\left\|U^{q-1} \partial_{i} U\right\|_{L^{\infty}\left(B_{3 r / 4}\right)}\right) \\
& \leq C\left(1+\left\|U^{q-1}\right\|_{L^{\infty}\left(B_{r}\right)}\right)\|U\|_{L^{\infty}\left(Q_{r}\right)} .
\end{aligned}
$$

Next, by employing Lemma 8.2 (2), we obtain the second inequality, i.e.,

$$
\left\|t^{1-2 s} \partial_{t} U\right\|_{C^{\alpha}\left(Q_{r / 2}\right)} \leq C\left(\|U\|_{L^{\infty}\left(Q_{r}\right)}+\left\|U^{q}\right\|_{C^{\beta}\left(B_{r}\right)}\right) .
$$

Besides, an application of Lemma 8.1 to (8.3) as well as inequality (8.4) imply that

$$
\begin{aligned}
\left\|\partial_{i} \partial_{j} U\right\|_{L^{\infty}\left(Q_{3 r / 4}\right)} & \leq C\left(\int_{Q_{4 r / 5}} t^{1-2 s}\left|\partial_{i} \partial_{j} U\right|^{2} d z\right)^{1 / 2}+C\left\|U^{q-2}\left(\partial_{i} U\right)\left(\partial_{j} U\right)\right\|_{L^{\infty}\left(B_{4 r / 5}\right)} \\
& \leq C\left(\|U\|_{L^{\infty}\left(Q_{r}\right)}+\left\|U^{q-2}\left(\partial_{i} U\right)\left(\partial_{j} U\right)\right\|_{L^{\infty}\left(B_{r}\right)}\right) .
\end{aligned}
$$

Therefore, Lemma 8.2 (1) shows

$$
\begin{aligned}
\left\|\partial_{i} \partial_{j} U\right\|_{C^{\alpha}\left(Q_{r / 2}\right)} \\
\leq C\left(\left\|\partial_{i} \partial_{j} U\right\|_{L^{\infty}\left(Q_{3 r / 4}\right)}+\left\|U^{q-1} \partial_{i} \partial_{j} U\right\|_{L^{\infty}\left(B_{3 r / 4}\right)}+\left\|U^{q-2}\left(\partial_{i} U\right)\left(\partial_{j} U\right)\right\|_{L^{\infty}\left(B_{3 r / 4}\right)}\right) \\
\leq C\left(\|U\|_{L^{\infty}\left(Q_{r}\right)}+\left\|U^{q-2}\left(\partial_{i} U\right)\left(\partial_{j} U\right)\right\|_{L^{\infty}\left(B_{r}\right)}\right) \\
\quad+C\left\|U^{q-1}\right\|_{L^{\infty}\left(B_{r}\right)}\left(\|U\|_{L^{\infty}\left(Q_{r}\right)}+\left\|U^{q-2}\left(\partial_{i} U\right)\left(\partial_{j} U\right)\right\|_{L^{\infty}\left(B_{r}\right)}\right),
\end{aligned}
$$

which is the third inequality of Proposition 8.3. On the other hand, by employing Lemma 8.2 (2) to (8.2) again, we deduce the fourth inequality

$$
\left\|t^{1-2 s} \partial_{t} \partial_{i} U\right\|_{C^{\alpha}\left(Q_{r / 2}\right)} \leq C\left(\left\|\partial_{i} U\right\|_{L^{\infty}\left(Q_{r}\right)}+\left\|U^{q-1} \partial_{i} U\right\|_{L^{\infty}\left(B_{r}\right)}\right) .
$$

Finally, the last inequality follows from the fact that

$$
t^{2-2 s} \partial_{t}^{2} U=-(1-2 s) t^{1-2 s} \partial_{t} U-t^{2-2 s} \Delta_{x} U \text { in } Q_{2 r} .
$$

This completes the proof.

As a corollary of the above result, we get

Corollary 8.4 Let $\left\{U_{n}\right\}_{n \in \mathbb{N}}$ is a sequence of solutions of (2.4) with $\epsilon=\epsilon_{n}$. For any $r>0$, let $A_{r}^{\prime}=\mathcal{C}^{\prime} \backslash \cup_{i=1}^{m} \overline{B_{+}^{N+1}\left(\left(x_{0}^{i}, 0\right), r\right)}$. Then, there exist $\alpha \in(0,1)$ and a constant $C>0$ independent of $n \in \mathbb{N}$ such that

$$
\sum_{k=1}^{2}\left\|\nabla_{x}^{k}\left(\left(\lambda_{n}^{1}\right)^{-\frac{N-2 s}{2}} U_{n}\right)\right\|_{C^{\alpha}\left(A_{r}^{\prime}\right)}+\sum_{\substack{0 \leq k \leq 1,1 \leq l \leq 2, 1 \leq k+l \leq 2}}\left\|t^{l-2 s} \partial_{t}^{l} \nabla_{x}^{k}\left(\left(\lambda_{n}^{1}\right)^{-\frac{N-2 s}{2}} U_{n}\right)\right\|_{C^{\alpha}\left(A_{r}^{\prime}\right)} \leq C
$$

for any $n \in \mathbb{N}$ large enough.

Proof Fix any compact subset $K \subset A_{r}^{\prime}$ such that $K \cap \Omega \neq \varnothing$. By (4.21), we have $\left\|U_{n}\right\|_{L^{\infty}(K)} \leq C\left(\lambda_{n}^{1}\right)^{\frac{N-2 s}{2}}$ (cf. Lemma 4.4). Since Green's function $G_{\mathcal{C}}$ is positive in $\mathcal{C}$, again (4.21) tells us that the value $\inf _{z \in K}\left(\lambda_{n}^{1}\right)^{-\frac{N-2 s}{2}} U_{n}(z)$ is bounded away from zero for large $n \in \mathbb{N}$. Thus, even in the case that $p-2-\epsilon_{n}=\frac{6-N}{N-2}-\epsilon_{n}<0$ (i.e., $N \geq 6$ ), we know

$$
\left\|U^{p-2-\epsilon_{n}}\left|\nabla_{x} U\right|^{2}\right\|_{L^{\infty}\left(B_{r}\right)} \leq C\left(\lambda_{n}^{1}\right)^{\left(\frac{N-2 s}{2}\right)\left(p-\epsilon_{n}\right)} .
$$


As a consequence,

$$
\sum_{k=1}^{2}\left\|\nabla_{x}^{k} U_{n}\right\|_{C^{\alpha}\left(A_{r}^{\prime}\right)}+\sum_{\substack{0 \leq k \leq 1,1 \leq l \leq 2, 1 \leq k+l \leq 2}}\left\|t^{l-2 s} \partial_{t}^{l} \nabla_{x}^{k} U_{n}\right\|_{C^{\alpha}\left(A_{r}^{\prime}\right)} \leq C\left(\lambda_{n}^{1}\right)^{\frac{N-2 s}{2}} .
$$

The proof is finished.

Proof of (4.22) and (4.23) Let us consider the sequence $\left\{\nabla_{x} U_{n}\right\}_{n \in \mathbb{N}}$. By Corollary 8.4, it converges to some function $F$ uniformly over a compact subset of $A_{r}^{\prime}$. Then (4.21) and an elementary analysis imply the fact that $F=c_{1} \sum_{i=1}^{m} b_{i} \nabla_{x} G_{\mathcal{C}}\left((x, t), x_{0}^{i}\right)$. The other functions can be treated similarly. This proves (4.22) and (4.23).

\section{References}

1. Abdelhedi, W., Chtioui, H.: On a Nirenberg-type problem involving the square root of the Laplacian. J. Funct. Anal. 265, 2937-2955 (2013)

2. Almaraz, S.: The asymptotic behavior of Palais-Smale sequences on manifolds with boundary. Pac. J. Math. 269, 1-17 (2014)

3. Ao, W., Wei, J. Yang, W.: Infinitely many positive solutions of a fractional nonlinear Schrödinger equation with non-symmetric potentials (preprint)

4. Bahri, A., Coron, J.M.: On a nonlinear elliptic equation involving the critical Sobolev exponent: the effect of the topology of the domain. Commun. Pure Appl. Math. 41, 253-294 (1988)

5. Bahri, A., Li, Y.Y., Rey, O.: On a variational problem with lack of compactness: the topological effect of the critical points at infinity. Calc. Var. Partial Differ. Equ. 3, 67-93 (1995)

6. Barrios, B., Colorado, E., de Pablo, A., Sánchez, U.: On some critical problems for the fractional Laplacian operator. J. Differ. Equ. 252, 6133-6162 (2012)

7. Barrios, B., Colorado, E., Servadei, R., Soria, F.: A critical fractional equation with concave-convex power nonlinearities. Ann. Inst. H. Poincaré Anal. Non Linéaire 22, 875-900 (2015)

8. Berestycki, H., Coulon, A., Roquejoffre, J., Rossi, L.: The effect of a line with nonlocal diffusion on Fisher-KPP propagation. Math. Models Methods Appl. Sci. 25, 2519-2562 (2015)

9. Bonforte, M., Sire, Y., Vazquéz, J.-L.: Existence, uniqueness and asymptotic behaviour for fractional porous medium equations on bounded domains. Discrete Contin. Dyn. Syst. 35, 5725-5767 (2015)

10. Brändle, C., Colorado, E., de Pablo, A., Sánchez, U.: A concave-convex elliptic problem involving the fractional Laplacian. Proc. R. Soc. Edinb. Sect. A 143, 39-71 (2013)

11. Cabré, X., Roquejoffre, J.: The influence of fractional diffusion in Fisher-KPP equations. Commun. Math. Phys. 320, 679-722 (2013)

12. Cabré, X., Sire, Y.: Nonlinear equations for fractional Laplacians, I: Regularity, maximum principles, and Hamiltonian estimates. Ann. Inst. H. Poincaré Anal. Non Linéaire 31, 23-53 (2014)

13. Cabré, X., Sire, Y.: Nonlinear equations for fractional Laplacians II: existence, uniqueness, and qualitative properties of solutions. Trans. Am. Math. Soc. 367, 911-941 (2015)

14. Cabré, X., Tan, J.: Positive solutions of nonlinear problems involving the square root of the Laplacian. Adv. Math. 224, 2052-2093 (2010)

15. Caffarelli, L.A., Silvestre, L.: An extension problem related to the fractional Laplacian. Comm. Partial Differ. Equ. 32, 1245-1260 (2007)

16. Caffarelli, L.A., Stinga, P.R.: Fractional elliptic equations, Caccioppoli estimates and regularity. Ann. Inst. H. Poincaré Anal. Non Linéaire. doi:10.1016/j.anihpc.2015.01.004

17. Capella, A., Dávila, J., Dupaigne, L., Sire, Y.: Regularity of radial extremal solutions for some non-local semilinear equations. Commun. Partial Differ. Equ. 36, 1353-1384 (2011)

18. Carlen, E.A., Loss, M.: Extremals of functionals with competing symmetries. J. Funct. Anal. 88, 437-456 (1990)

19. Chang, S.-Y., González, M.: Fractional Laplacian in conformal geometry. Adv. Math. 226, 1410-1432 (2011)

20. Chen, W., Li, C., Ou, B.: Classification of solutions for an integral equation. Commun. Pure Appl. Math. 59, 330-343 (2006)

21. Chen, C.C., Lin, C.S.: Estimates of the conformal scalar curvature equation via the method of moving planes. II. J. Differ. Geom. 49, 115-178 (1998) 
22. Chen, G., Zheng, Y.: Concentration phenomenon for fractional nonlinear Schrodinger equations. Commun. Pure Appl. Anal. 13, 2359-2376 (2014)

23. Choi, W.: On strongly indefinite systems involving the fractional Laplacian. Nonlinear Anal. 120, 127-153 (2015)

24. Choi, W., Kim, S.: On perturbations of the fractional Yamabe problem. (preprint) arXiv:1501.00641

25. Choi, W., Kim, S., Lee, K.-A.: Asymptotic behavior of solutions for nonlinear elliptic problems with the fractional Laplacian. J. Funct. Anal. 266, 6531-6598 (2014)

26. Choi, W., Kim, S., Lee, K.-A.: Qualitative properties of multi-bubble solutions for nonlinear elliptic equations involving critical exponents. Adv. Math. (to appear)

27. Choi, W., Seok, J.: Infinitely many solutions for semilinear nonlocal elliptic equations under noncompact settings. (preprint) arXiv:1404.1132

28. Dávila, J., del Pino, M., Wei, J.: Concentrating standing waves for the fractional nonlinear Schrödinger equations. J. Differ. Equ. 256, 858-892 (2014)

29. Dávila, J., López, L., Sire, Y.: Bubbling solutions for nonlocal elliptic problems. (preprint) arXiv: 1410.5461

30. Fall, M.M., Weth, T.: Nonexistence results for a class of fractional elliptic boundary value problems. J. Funct. Anal. 263, 2205-2227 (2012)

31. Fang, Y., González, M. D.: Asymptotic behavior of Palais-Smale sequences associated with fractional Yamabe type equations. Pac. J. Math. 278, 369-405 (2015)

32. Felmer, P., Quaas, A., Tan, J.: Positive solutions of the nonlinear Schrdinger equation with the fractional Laplacian. Proc. R. Soc. Edinb. Sect. A 142, 1237-1262 (2012)

33. Frank, R.L., Lieb, E.H.: Inversion positivity and the sharp Hardy-Littlewood-Sobolev inequality. Calc. Var. Partial Differ. Equ. 39, 85-99 (2010)

34. González, M., Qing, J.: Fractional conformal Laplacians and fractional Yamabe problems. Anal. PDE 6, 1535-1576 (2013)

35. González, M., Wang, M.: Further results on the fractional Yamabe problem: the umbilic case. (preprint) arXiv: 1503.02862

36. Grossi, M., Pacella, F.: On an eigenvalue problem related to the critical exponent. Math. Z. 250, 225-256 (2005)

37. Grubb, G.: Regularity of spectral fractional Dirichlet and Neumann problems. J. Math. Nachr. (2015). doi:10.1002/mana.201500041

38. Han, Z.-C.: Asymptotic approach to singular solutions for nonlinear elliptic equations involving critical Sobolev exponent. Ann. Inst. H. Poincaré Anal. Non Linéaire 8, 159-174 (1991)

39. Jin, T., Li, Y.Y., Xiong, J.: On a fractional Nirenberg problem, part I: blow up analysis and compactness of solutions. J. Eur. Math. Soc. 16, 1111-1171 (2014)

40. Jin, T., Li, Y.Y., Xiong, J.: On a fractional Nirenberg problem, Part II: existence of Solutions. Int. Math. Res. Notices 2015, 1555-1589 (2015)

41. Kim, S., Musso, M., Wei, J.: A non-compactness result on the fractional Yamabe problem in large dimensions. (preprint) arXiv:1505.06183

42. Li, Y.Y.: Remark on some conformally invariant integral equations: the method of moving spheres. J. Eur. Math. Soc. 6, 153-180 (2004)

43. Li, Y.Y., Zhang, L.: Compactness of solutions to the Yamabe problem. II. Calc. Var. Partial Differ. Equ. 24, 185-237 (2005)

44. Li, Y.Y., Zhu, M.: Uniqueness theorems through the method of moving spheres. Duke Math. J. 80, 383-417 (1995)

45. Lieb, E.H.: Sharp constants in the Hardy-Littlewood-Sobolev and related inequalities. Ann. Math. 118, 349-374 (1983)

46. Musina, R., Nazarov, A.I.: On fractional Laplacians. Commun. Partial Differ. Equ. 39, 1780-1790 (2014)

47. Musso, M., Pistoia, A.: Multispike solutions for a nonlinear elliptic problem involving the critical Sobolev exponent. Indiana Univ. Math. J. 51, 541-579 (2002)

48. Palatucci, G., Pisante, A.: A global compactness type result for Palais-Smale sequences in fractional Sobolev spaces. Nonlinear Anal. 117, 1-7 (2015)

49. Rey, O.: The role of the Green's function in a nonlinear elliptic equation involving the critical Sobolev exponent. J. Funct. Anal. 89, 1-52 (1990)

50. Rey, O.: Blow-up points of solutions to elliptic equations with limiting nonlinearity. Differ. Integral Equ. 4, 1155-1167 (1991)

51. Rey, O.: The topological impact of critical points at infinity in a variational problem with lack of compactness: The dimension 3. Adv. Differ. Equ. 4, 581-616 (1999)

52. Ros-Oton, X., Serra, J.: The Dirichlet problem for the fractional Laplacian: regularity up to the boundary. J. Math. Pure Appl. 101, 275-302 (2014) 
53. Ros-Oton, X., Serra, J.: The extremal solution for the fractional Laplacian. Calc. Var. Partial Differ. Equ. 50, 723-750 (2014)

54. Ros-Oton, X., Serra, J.: The Pohozaev identity for the fractional Laplacian. Arch. Ration. Mech. Anal. 213, 587-628 (2014)

55. R. Schoen: Topics of differential geometry, given at Stanford University and New York University (19881989). https://www.math.washington.edu/pollack/research/Schoen-1988-notes.html

56. Servadei, R., Valdinoci, E.: On the spectrum of two different fractional operators. Proc. R. Soc. Edinb. Sect. A 144, 831-855 (2014)

57. Servadei, R., Valdinoci, E.: The Brezis-Nirenberg result for the fractional Laplacian. Trans. Am. Math. Soc. 367, 67-102 (2015)

58. Stinga, P.R., Torrea, J.L.: Extension problem and Harnack's inequality for some fractional operators. Commun. Partial Differ. Equ. 35, 2092-2122 (2010)

59. Struwe, M.: A global compactness result for elliptic boundary value problems involving limiting nonlinearities. Math. Z. 187, 511-517 (1984)

60. Tan, J.: The Brezis-Nirenberg type problem involving the square root of the Laplacian. Calc. Var. Partial Differ. Equ. 42, 21-41 (2011)

61. Tan, J.: Positive solutions for non local elliptic problems. Discrete Contin. Dyn. Syst. 33, 837-859 (2013) 